\title{
Are Sudden Stratospheric Warmings Preceded by Anomalous Tropospheric Wave Activity? 0
}

\author{
Alvaro DE LA CÁMARA \\ Department of Earth Physics and Astrophysics, Universidad Complutense de Madrid, and Institute of Geosciences, \\ Centro Mixto del Consejo Superior de Investigaciones Científicas (CSIC) and Universidad Complutense de Madrid, \\ Madrid, Spain \\ THOMAS BIRNER \\ Meteorological Institute, Ludwig-Maximilians-Universität München, Munich, Germany \\ JOHN R. ALBERS \\ Cooperative Institute for Research in Environmental Sciences, University of Colorado Boulder, and Physical Sciences \\ Division, NOAA/Earth System Research Laboratory, Boulder, Colorado
}

(Manuscript received 6 April 2019, in final form 22 July 2019)

\begin{abstract}
A combination of 240 years of output from a state-of-the-art chemistry-climate model and a twentiethcentury reanalysis product is used to investigate to what extent sudden stratospheric warmings are preceded by anomalous tropospheric wave activity. To this end we study the fate of lower tropospheric wave events (LTWEs) and their interaction with the stratospheric mean flow. These LTWEs are contrasted with sudden stratospheric deceleration events (SSDs), which are similar to sudden stratospheric warmings but place more emphasis on the explosive dynamical nature of such events. Reanalysis and model output provide very similar statistics: Around one-third of the identified SSDs are preceded by wave events in the lower troposphere, while two-thirds of the SSDs are not preceded by a tropospheric wave event. In addition, only $20 \%$ of all anomalous tropospheric wave events are followed by an SSD in the stratosphere. This constitutes statistically robust evidence that the anomalous amplification of wave activity in the stratosphere that drives SSDs is not necessarily due to an anomalous amplification of the waves in the source region (i.e., the lower troposphere). The results suggest that the dynamics in the lowermost stratosphere and the vortex geometry are essential, and should be carefully analyzed in the search for precursors of SSDs.
\end{abstract}

\section{Introduction}

Sudden stratospheric warmings (SSWs) are major disruptions of the wintertime stratospheric polar vortex of the Northern Hemisphere (Labitzke 1977; Butler et al. 2015), which profoundly alter the stratospheric circulation and transport at a global scale (see de la Cámara et al. 2018a,b, and references therein). Perhaps the most important impact of SSWs in terms of potential societal repercussions is their influence on Euro-Atlantic

\footnotetext{
Supplemental information related to this paper is available at the Journals Online website: https://doi.org/10.1175/JCLI-D-190269.s1.

Corresponding author: Alvaro de la Cámara, acamarai@ucm.es
}

weather regimes: the associated stratospheric temperature and wind anomalies during SSWs can induce changes in the storm track that impact surface weather for up to two months (Baldwin and Dunkerton 2001; Thompson et al. 2002; Kidston et al. 2015; Ayarzagüena et al. 2018). Indeed, increasing evidence suggests that SSWs are a source of additional predictability in subseasonal-toseasonal forecasting of winter climate in Europe and eastern North America (Marshall and Scaife 2010; Smith and Kushner 2012; Tripathi et al. 2015; Scaife et al. 2016; Butler et al. 2019).

In this context, understanding the still unclear mechanisms behind the explosive growth of stratospheric wave activity that ultimately triggers SSWs arises as a major scientific question. In particular, much of the discussion centers on the relative roles of tropospheric 
and stratospheric processes in the wave flux amplification that happens in the stratosphere. On one hand, the planetary Rossby waves present in the stratosphere have their primary sources in the troposphere (Andrews et al. 1987). Therefore, an often-invoked explanation for the rapid increase of stratospheric wave fluxes is the anomalous excitation of these waves in the troposphere and their subsequent propagation into the stratosphere (e.g., Matsuno 1971; Polvani and Waugh 2004). In this context, several phenomena have been linked with an increased probability of SSW occurrence, such as El NiñoSouthern Oscillation (ENSO), the Madden-Julian oscillation (MJO), blockings, and so on (e.g., Martius et al. 2009; Butler and Polvani 2011; Garfinkel et al. 2012; Scaife et al. 2014; Barriopedro and Calvo 2014; Colucci and Kelleher 2015; Domeisen et al. 2018a; Garfinkel et al. 2018). On the other hand, stratospheric conditions may crucially determine the occurrence of an SSW by modulating the propagation of wave activity from the troposphere, developing its own internal instabilities, or even favoring resonant wave amplification (e.g., Clark 1974; Plumb 1981; McIntyre 1982; Chen and Robinson 1992; Christiansen 1999; Esler and Scott 2005; Domeisen et al. 2018a). Experiments with highly truncated stratosphereonly models (i.e., with an inactive troposphere) have suggested that the stratosphere is able to internally generate oscillations that resemble SSWs (e.g., Holton and Mass 1976; Yoden 1987; Scott and Haynes 2000; Chen et al. 2001; Matthewman and Esler 2011; Esler and Matthewman 2011). In particular, Sjoberg and Birner (2014) have demonstrated the ability of such a model stratosphere to internally generate an SSW even with constant prescribed bottom boundary wave activity flux. But to what extent is this applicable to more complex global models, or even to reality, where there is strong transient forcing from the troposphere? Results from simulations with three-dimensional primitive equation models of the stratosphere (Smith 1989, 1992; Martineau et al. 2018a), global dry dynamical core models (Scott and Polvani 2004; Hitchcock and Haynes 2016; Jucker 2016; Martineau et al. 2018a; Lindgren et al. 2018), and state-ofthe-art general circulation models (Christiansen 1999; Scott and Polvani 2006; de la Cámara et al. 2017) point out the ability of the stratosphere to modulate or control the occurrence of, and even internally generate, SSWs.

There are also studies that use reanalysis products to gain new insights into the relative roles of anomalous tropospheric wave injection and stratospheric control on the explosive growth of stratospheric wave activity that triggers SSWs. In particular, Birner and Albers (2017, hereafter BA17) used 38 years of reanalysis fields to reveal that only about $33 \%$ of the observed SSWs since 1979 were preceded by anomalously strong lower tropospheric wave events, and that only $20 \%$ of the lower tropospheric wave events were followed by SSWs. These results give context to recent case studies that have provided evidence of the essential role of the stratosphere state (i.e., vortex preconditioning) in the development of the SSWs that occurred in 2009 (Albers and Birner 2014; Domeisen et al. 2018b) and 2013 (Attard et al. 2016).

The goal of the present paper is to expand the study of BA17 and to investigate if SSWs are preceded by anomalous tropospheric wave activity in long climate records. We will use historical climate simulations of the Whole Atmosphere Community Climate Model (WACCM) and data from the ECMWF twentieth-century reanalysis (ERA-20C). We identify lower tropospheric events of anomalously strong wave activity, as well as midstratospheric events of strong zonal wind deceleration. In agreement with previous findings (BA17; White et al. 2019), our results show that only $\sim 1 / 5$ of tropospheric wave events are followed by such stratospheric events, and that about $1 / 3$ of stratospheric events are preceded by tropospheric wave events. Composite analyses of the upward wave activity flux provide evidence that the rapid amplification of stratospheric wave fluxes that drives SSWs can hardly be attributed to amplification in the tropospheric wave sources. Analysis of vortex moment diagnostics suggests that the vortex is preferentially wider and centered over the pole before stratospheric events dominated by zonal wavenumber- 1 amplification.

The remainder of the paper is organized as follows. Section 2 describes the model output, methods, and diagnostics employed, while section 3 presents the results. A summary and discussion of the main results are included in section 4 .

\section{Data and methods}

\section{a. Model output and reanalysis}

We use output from a state-of-the-art chemistry climate model and a twentieth-century reanalysis. The model used is the Whole Atmosphere Community Climate Model (WACCM) version 4 (Marsh et al. 2013; Garcia et al. 2017), which can serve as the atmospheric component of the Community Earth System Model developed at the National Center for Atmospheric Research. We use an ensemble of four members (each member using slightly different initial conditions of the atmospheric state) forced with observed sea surface temperatures and external forcings for the period 1955-2014, with a horizontal resolution of $2.5^{\circ} \times 1.9^{\circ}$ longitude-latitude, and 66 levels in the vertical with the top at about $140-\mathrm{km}$ altitude. This provides 240 years of daily output. 
We also use daily meteorological fields from the reanalysis of the twentieth century ERA-20C (Poli et al. 2016), developed at the European Centre for MediumRange Weather Forecasts (ECMWF). The horizontal resolution is approximately $125 \mathrm{~km}$ and has 91 vertical levels between the surface and $0.01 \mathrm{hPa}$. In particular we use the zonal-mean dataset described in Martineau et al. (2018b). ERA-20C covers the period 1900-2010, assimilating observations of surface pressure and surface winds over the ocean. It is important to remark that ERA-20C is not taken in this study as a reliable reproduction of the synoptic evolution of the stratosphere (previous studies have shown that ERA-20C presents a reasonable stratospheric variability; see Gerber and Martineau 2018; Hitchcock 2019), but rather as a valuable long dataset from which statistics can be extracted.

To calculate daily anomalies of the different fields, we use a slowly evolving climatological seasonal cycle, which is computed as follows. Starting from monthlymean averages, we first calculate $30-y r$ means centered on every 10th year for each individual month, and then we interpolate back to a yearly time series (for each individual month). Finally, we interpolate the monthlymean climatology to a daily time series, producing a daily seasonal cycle that smoothly varies over the years. Deseasonalized anomalies are then calculated as the difference between the original daily fields and the seasonal cycle, after which a 10-day running mean is applied.

Unless stated otherwise, anomalies are normalized by the daily standard deviation $\sigma$ (referred to as standardized anomalies throughout the paper). This provides a measure of how far is an anomaly from the climatological mean in $\sigma$ units and, therefore, gives a continuous measure of statistical significance. ${ }^{1}$ In terms of a Student's $t$ test, essentially all anomalies shown in the composites in section 3 are statistically significant at the $95 \%$ confidence level.

\section{b. Identification of events}

We have used similar methods to those of BA17 for the identification of anomalous lower troposphere wave events (LTWEs), employing time series of standardized anomalies of the vertical component of

\footnotetext{
${ }^{1}$ The $t$ statistic in a Student's $t$ test for the significance of a composite mean is approximately proportional to the standardized anomaly and the square root of the degrees of freedom, which in this study is the number of events (tropospheric wave events or sudden warmings) minus one. This means that threshold values for the statistical significance at a given confidence level are approximately parallel to isopleths of standardized anomalies.
}

the Eliassen-Palm flux $\left(F_{z}\right)$ (Andrews et al. 1987) at $600 \mathrm{hPa}$ averaged over $45^{\circ}-75^{\circ} \mathrm{N}$. Since SSWs in the stratosphere tend to be triggered by amplification of individual planetary waves, and previous studies have reported a high degree of anticorrelation between the longest planetary waves (e.g., Labitzke 1978, 1981), this is done separately for the zonal harmonics with wavenumber $1\left[F_{z}(s=1)\right]$ and $2\left[F_{z}(s=2)\right]$. The selection of a different pressure level in the lower troposphere does not affect the results significantly (not shown). We identify an LTWE when the standardized anomaly of $F_{z}$ exceeds the value of $2 \sigma$. The central date of the event is taken as the day with the maximum value of $F_{z}$, and consecutive events must be at least 20 days apart, in a manner consistent with the definition of SSWs and that is approximatively two radiative relaxation time scales of the polar midstratosphere (Newman and Rosenfield 1997; Charlton and Polvani 2007). With these criteria, there are 191 LTWE1 and 182 LTWE2 in WACCM, and 71 and 91 in ERA-20C, respectively.

For SSWs, we use a metric based on the deceleration of the zonal mean wind at $60^{\circ} \mathrm{N}$ and $10 \mathrm{hPa}$, and thus are referred to as sudden stratospheric deceleration (SSD) events. An SSD is identified when the 10-day change in the zonal mean zonal wind falls below $-18 \mathrm{~m} \mathrm{~s}^{-1}$ in WACCM and $-20 \mathrm{~m} \mathrm{~s}^{-1}$ in ERA-20C (which approximately equal 2 standard deviations in each dataset), with the central date set on the day with the strongest deceleration. Again, two consecutive events must be separated by at least 20 days. This procedure is similar to that described in Martineau and Son (2015) and sets the focus on the dynamical event. We identify 200 SSDs in WACCM and 95 in ERA-20C.

We further classify SSDs according to the dominant planetary wavenumber during the event. Taking the standardized anomalies of $F_{z}$ at $50 \mathrm{hPa}$, a wave-1 SSD (SSD1) is classified if $F_{z}(s=1)$ is larger than $2 \sigma$ in any day within \pm 5 days from the SSW central date, provided that $F_{z}(s=2)$ is smaller than $2 \sigma$. Conversely, a wave-2 SSD (SSD2) is classified if $F_{z}(s=2)$ is larger than, and $F_{z}(s=1)$ is smaller than, $2 \sigma$. If both $F_{z}(s=1)$ and $F_{z}(s=2)$ meet the $2 \sigma$ threshold any day within \pm 5 days from the central date, the event is classified as a wave- 1 and 2 event (SSD1\&2). The rest of the events, where neither $F_{z}(s=1)$ or $F_{z}(s=2)$ meet the threshold, are referred to as weak wave SSDs (SSDww).

\section{c. Vortex moment diagnostics}

In this study we make use of vortex moments and elliptical diagnostics (Melander et al. 1986; Waugh 1997) to extract time series of the centroid position $\left(\lambda_{c}, \phi_{c}\right)$, area $A$, and aspect ratio $r$ of the equivalent ellipse. The 
"equivalent ellipse" is an ellipse of uniform potential vorticity (PV) with the same moment diagnostic as the polar vortex at a given time. For this purpose we use daily mean values of $\mathrm{PV}$ at the isentropic surface of $850 \mathrm{~K}(\sim 10 \mathrm{hPa})$ from both WACCM and ERA-20C, and apply the methodology of Matthewman et al. (2009) to compute both the absolute vortex moments $M_{k l}$

$$
M_{k l}=\iint\left[\hat{q}(x, y)-q_{b}\right] x^{k} y^{l} d x d y
$$

and the relative vortex moments $J_{k l}$

$$
J_{k l}=\iint\left[\hat{q}(x, y)-q_{b}\right]\left(x-x_{c}\right)^{k}\left(y-y_{c}\right)^{l} d x d y
$$

In Eqs. (1) and (2) $q$ is $\mathrm{PV}, q_{b}$ is the vortex boundary taken as the PV average north of $45^{\circ} \mathrm{N}$, and $\hat{q}$ is a modified PV field equal to $q$ if $q>q_{b}$ (i.e., in the vortex interior), and equal to $q_{b}$ elsewhere. Note that we have followed Waugh (1997) in the selection of the value of the vortex boundary $q_{b}$, and that it was shown in that study that the results were not sensitive to this selection. Also, $(x, y)$ are coordinates in the Lambert's azimuthal projection, $(k, l)$ give the moments order in the $(x, y)$ directions, and $\left(x_{c}, y_{c}\right)$ are the position of the ellipse's centroid defined as

$$
\left(x_{c}, y_{c}\right)=\frac{1}{M_{00}}\left(M_{10}, M_{01}\right) .
$$

With these definitions, the area of the equivalent ellipse is computed as

$$
A=\frac{M_{00}}{q_{b}},
$$

and the ellipse's aspect ratio as

$$
r=\left|\frac{J_{20}+J_{02}+\sqrt{4 J_{11}^{2}+\left(J_{20}-J_{02}\right)^{2}}}{J_{20}+J_{02}-\sqrt{4 J_{11}^{2}+\left(J_{20}-J_{02}\right)^{2}}}\right|^{1 / 2} .
$$

The ellipse centroid position [Eq. (3)] gives a measure of the displacement of the vortex, the ellipse area [Eq. (4)] is an estimation of the vortex's area and strength, and the aspect ratio [Eq. (5)] is a measure of the vortex's elongation (i.e., the ratio between the major and minor axes of the vortex). The reader is referred to Matthewman et al. (2009) for further details.

\section{The relation between lower tropospheric wave events and sudden stratospheric warmings}

\section{a. Are extreme lower tropospheric wave events followed by SSDs?}

The first question we address is the fate of anomalously strong lower tropospheric wave events, so we bin the events according to whether or not they are followed by SSDs in the stratosphere in a time span of 0 to 10 days. This time scale of 10 days was found by Sjoberg and Birner (2012) to be associated with the wave forcing of SSWs. Group velocity estimates also motivate this time scale (see Albers and Birner 2014 and BA17), but note that the results are not very sensitive to slight variations in the length of this time scale. Table 1 presents the corresponding statistics. The results are remarkably similar between WACCM and ERA-20C; only $19 \%$ of all LTWEs are followed by SSDs in the climate model, and $17 \%$ in the reanalysis. Viewed as a function of the individual wavenumber events, $26 \%$ of LTWE1 events in WACCM and $30 \%$ in ERA-20C are followed by SSDs; for LTWE2, $12 \%$ and $8 \%$ of the events in the respective datasets are followed by SSDs.

Figure 1 shows the evolution of standardized anomalies of $F_{z}$ (black contours) and zonal wind acceleration (color shading), composited around LTWE1 and LTWE2 followed and not by SSDs in WACCM. By construction, the four composites show a peak in upward wave activity anomalies at $600 \mathrm{hPa}$ at lag 0 days (Figs. 1a,b,d,e), with values larger than $2 \sigma$. In the stratosphere, there are strong anomalies of wave activity and wind deceleration peaking at positive lags only in LTWE1 and LTWE2 events followed by SSDs (Figs. 1a,d). Peak values larger than $2 \sigma$ take place at lag 5 days simultaneously for both variables, but they are centered at $10-20 \mathrm{hPa}$ for wind deceleration and at around $100 \mathrm{hPa}$ for the wave activity. Also, the anomalies of wave activity span the whole depth of the stratosphere for LTWE1 (Fig. 1a), while they appear more restricted to the lower stratosphere for LTWE2 (Fig. 1d). For the events not followed by SSDs (Figs. 1b,e), the anomalies of wave activity in the stratosphere are smaller than $0.5 \sigma$, although there is a short period of time at lags 1-4 days with values over $1 \sigma$ in the lowermost stratosphere (below $100 \mathrm{hPa}$ ) for both LTWE1 and LTWE2. These (weaker) anomalies in stratospheric wave activity do not translate into changes of the stratospheric circulation, and the wind acceleration anomalies in the stratosphere remain small (shading in Figs. $1 \mathrm{~b}$ and 1e). Looking at the composite difference between events followed and not followed by SSDs (Figs. 1c,f), it is clear that the main differences are located in the stratosphere, particularly 
TABLE 1. Number of lower tropospheric wave events (LTWEs) followed and not followed by sudden stratospheric deceleration (SSD) events.

\begin{tabular}{llccr}
\hline \hline & & LTWE1 & LTWE2 & All LTWE \\
\hline WACCM & Followed by SSD & $50(26 \%)$ & $22(12 \%)$ & $72(19 \%)$ \\
& Not followed by SSD & $144(74 \%)$ & $157(88 \%)$ & $301(81 \%)$ \\
ERA-20C & Total & 194 & 179 & 373 \\
& Followed by SSD & $21(30 \%)$ & $7(8 \%)$ & $28(17 \%)$ \\
& Not followed by SSD & $50(70 \%)$ & $84(92 \%)$ & $134(83 \%)$ \\
\hline
\end{tabular}

for LTWE1 (note that the magenta lines and dots denote the areas with statistical significant differences of $F_{z}$ and wind acceleration, respectively, according to a Student's $t$ test). It is not the case that tropospheric wave events followed by SSDs are stronger than those not followed by SSDs.

Figure 2 shows the corresponding composites using ERA-20C. The structure and evolution of the anomalies are quite similar to those in WACCM (Fig. 1), especially for LTWE1 (top row). There are some differences between WACCM and ERA-20 in the composites corresponding to LTWE2 events followed by SSDs (although the number of events in ERA-20C is small) (Figs. 1d and 2d, respectively), such as a deeper extension of the $F_{z}(s=2)$ anomalies in the stratosphere in the case of ERA-20C. However, the main message extracted from Fig. 1 holds here: Sorting the tropospheric wave events regarding whether or not they are followed by SSDs does not discriminate the amplitude of wave activity anomalies in the troposphere. The main
WACCM

a) 50 LTWE1 / SSD

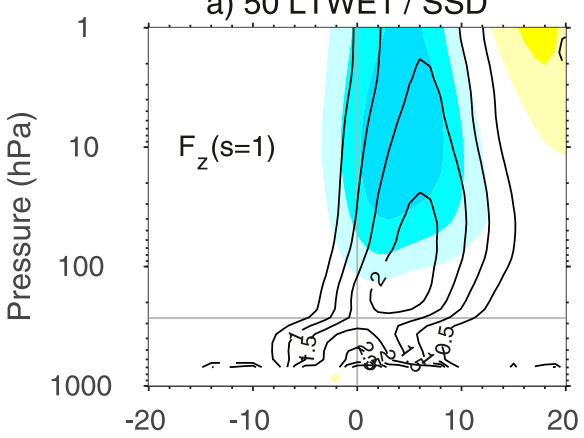

d) 22 LTWE2 / SSD

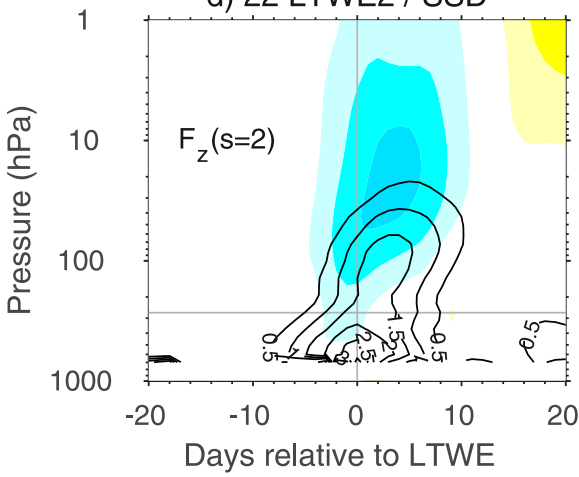

b) $144 \mathrm{LTWE} 1$ / no SSD

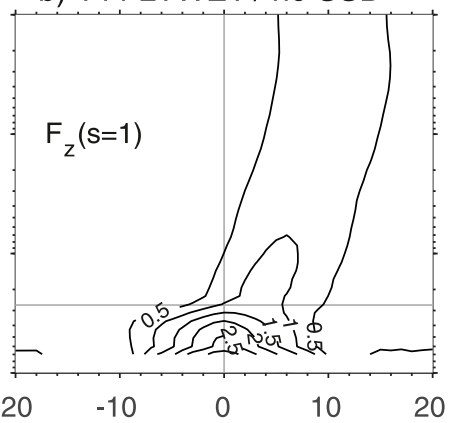

e) 157 LTWE2 / no SSD

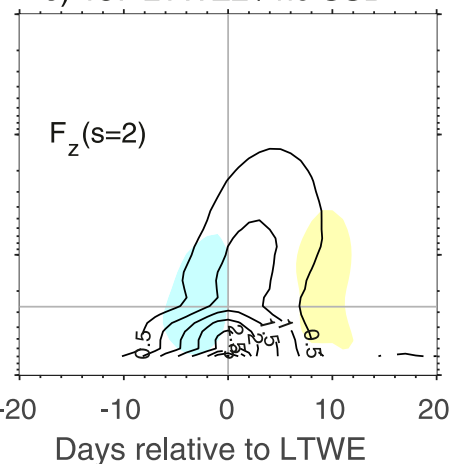

c) LTWE1 events difference

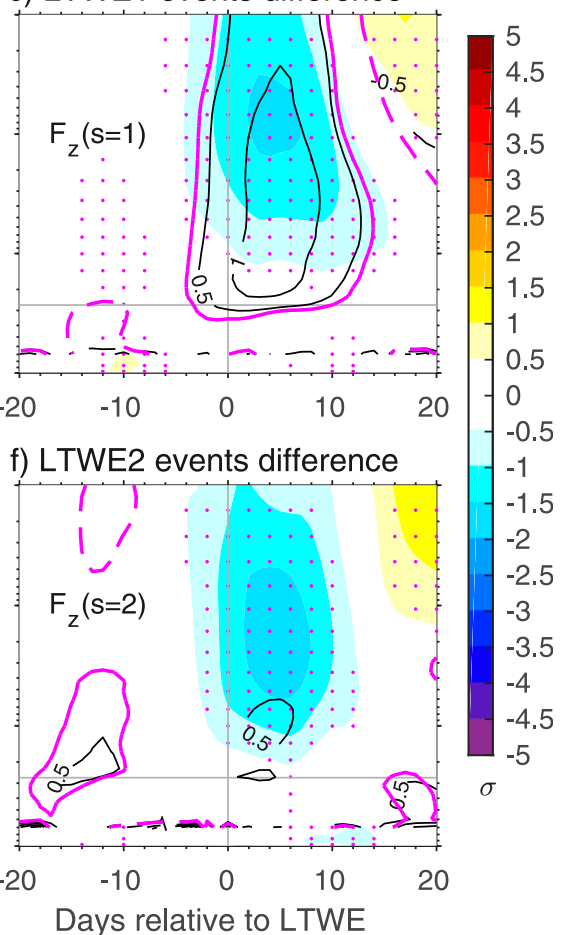

FIG. 1. Composite evolution, as a function of lag and pressure level, for extreme upward wave activity events (LTWEs) near $600 \mathrm{hPa}$ in WACCM. The upward EP flux anomaly $\left(F_{z}\right)$ is shown in black contours (quantized in individual zonal wavenumbers, as indicated), with the 10-day integrated wind tendency $\left(\partial_{t} U\right)$ in colors. Shown are (left) the subset of those LTWEs associated with an SSD, (middle) the subset of those LTWEs not associated with SSDs, and (right) the difference between the (left) and (middle), for (a)-(c) LTWE1 and (d)-(f) LTWE2. All anomalies are standardized (i.e., high values indicate high statistical significance). In (c) and (f), magenta lines and dots indicate statistically significant values for the composite differences of $F_{z}$ and $\partial_{t} U$, respectively, according to a two-tailed Student's $t$ test $(\alpha=0.05)$. A horizontal gray line marks the approximate tropopause level $(\sim 270 \mathrm{hPa})$. 
ERA-20C

a) 21 LTWE1 / SSD

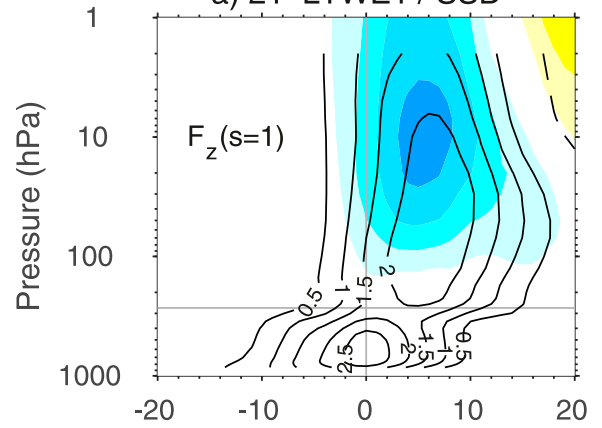

d) 7 LTWE2 / SSD

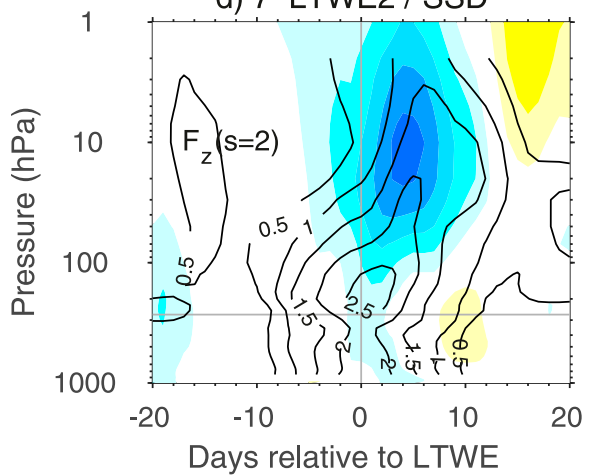

b) 50 LTWE 1 / no SSD

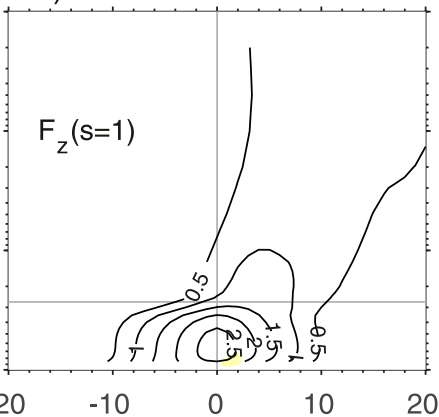

e) 84 LTWE 2 / no SSD

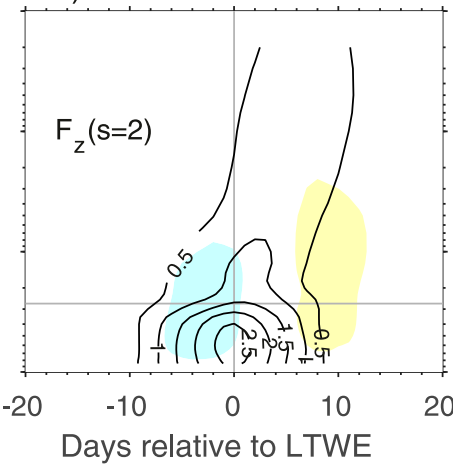

c) LTWE1 events difference

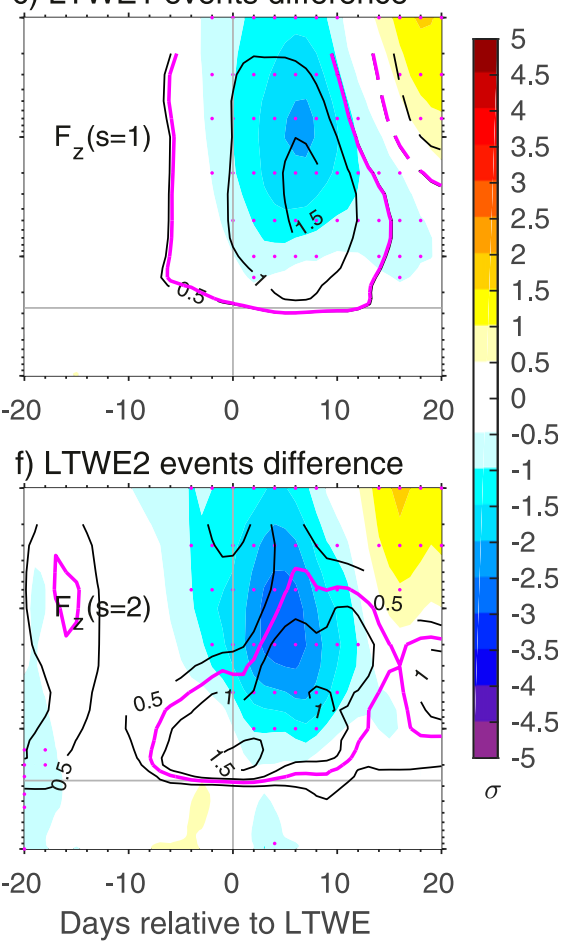

FIG. 2. As in Fig. 1, but for ERA-20C.

differences, as expected from the construction of the composites, happen in the stratosphere.

What is then the fate of the tropospheric wave activity when LTWEs are not followed by SSDs? Composites of EP flux diagrams on the central day of the event indicate a strong accumulation of wave activity around the tropopause in these cases (Figs. 3 and 4 for WACCM and ERA-20C, respectively). More importantly, the composite difference of LTWEs followed and not followed by SSDs shows no significant difference in wave propagation paths in the troposphere (Figs. 3c,f and $4 c, f)$. Instead, the difference of anomalous EP flux divergence (EPFD) shows positive values in the midlatitude upper troposphere/lower stratosphere; that is, anomalous convergence of wave activity in LTWEs not followed by SSDs with respect to those events followed by SSDs. Note that these results are consistent at different time lags around the central date of LTWEs (not shown).

BA17 suggested the presence of a wave source above the tropopause in LTWE1 events that are followed by SSDs, but they worked with only seven events of this type in ERA-Interim. Figure 5 displays the evolution of the 10-day running-mean EPFD around LTWEs at $\sim 250 \mathrm{hPa}$, in both WACCM (top row) and ERA-20C (bottom row), which have at least 3 times as many events. Note that this figure shows the net value of EPFD, not the anomalies. For LTWE1 followed by SSDs (red line in Figs. 5a and 5d), there is a transition from weak negative EPFD at negative lags to positive at positive lags, consistent with BA17's results. This transition in the sign of the EPFD could simply be the signature of wave propagation; as a wave packet propagates, it will be accompanied by EP flux convergence on the wave front (wave activity increasing) and by divergence behind (wave activity decreasing) (e.g., Hoskins 1983; Birner et al. 2013; Martineau and Son 2015). So in the presence of wave propagation, this signature on EPFD is expected to cancel upon time integration. However, the positive EPFD after the event is much larger than the negative EPFD before the event (and thus dominates the time average around the events) (Figs. 5a,d), so it can be interpreted as a net source of wave activity that contributes to the rapid growth of stratospheric wave activity in these cases (i.e., LTWE1 followed by SSDs). A much weaker transition from negative to positive EFFD happens in LTWE1 not followed by SSDs (Figs. 5b,e), and time integrating EPFD over the event clearly gives negative values in these cases (i.e., a wave activity sink). A possible explanation of a wave source above the tropopause is shear instability (Charney and Stern 1962). A necessary 

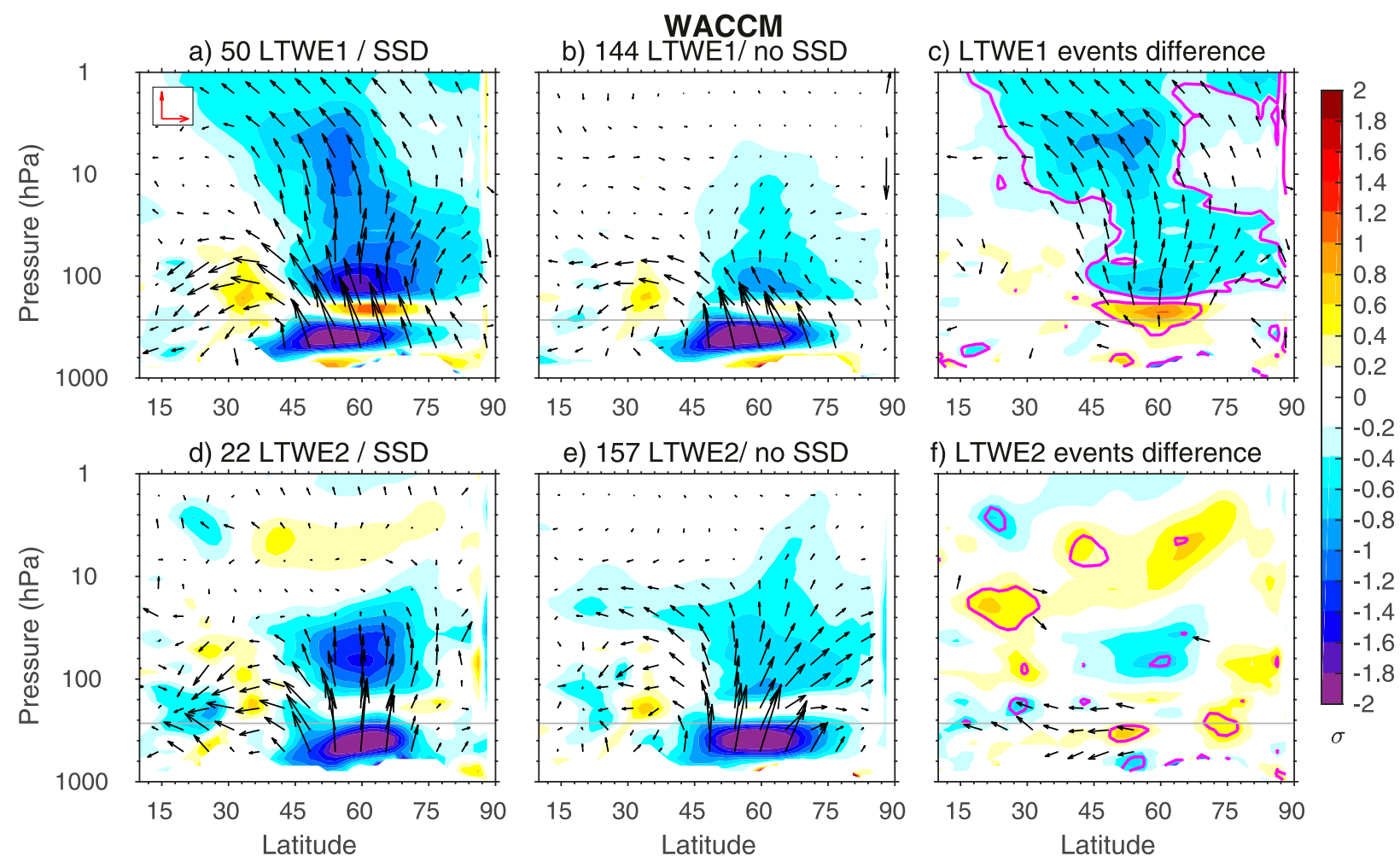

FIG. 3. EP flux diagrams for composites of LTWEs at lag 0 days, in WACCM. The meridional and vertical components of anomalies of the EP flux are shown in vectors, and the anomalous EP flux divergence (EPFD) is shown in colors. (left) Subset of those LTWEs associated with an SSD, (middle) subset of those LTWEs not associated with SSDs, and (right) difference between (left) and (middle), shown for (a)-(c) LTWE1, with variables plotted for zonal wave harmonic $s=1$, and (d)-(f) LTWE2, with variables plotted for zonal wave harmonic $s=2$. All values are standardized (i.e., high values indicate high statistical significance). The box in (a) shows the reference arrow length for a $1 \sigma$ anomaly. In (c) and (f), magenta lines indicate statistically significant values for the composite differences of EPFD, and vectors are plotted only if at least one component of the EP flux is statistically significant, according to a two-tailed Student's $t$ test $(\alpha=$ $0.05)$.

condition for barotropic/baroclinic instability is a reversal of the positive meridional potential vorticity gradient, but we have not found any signature of this necessary condition in our analysis (not shown). Another possibility for the wave-1 source above the tropopause is wave-wave interactions (Smith 1983; Smith et al. 1984); further research is needed to gain more insights into this wave-1 source.

Next we turn our attention to the evolution of the vortex geometry during LTWEs to better understand why some LTWEs are followed by SSDs while others (the majority) are not. The objective is to explore preconditioning ideas by which the state of the vortex may contribute to the wave activity burst in the stratosphere via modulation/focusing of wave propagation and/or favoring wave resonance (e.g., McIntyre 1982; Smith 1992). Figure 6 shows composite differences between LTWEs followed and not followed by SSDs of anomalies of the latitude of the vortex centroid (left panels), vortex area (middle panels), and vortex aspect ratio (right panels). In WACCM (upper panels of Fig. 6), the vortex centroid tends to be located $2^{\circ}$ to $4^{\circ}$ to the north, and the vortex tends to be larger and stronger, during the two weeks prior to LTWE1 when these are followed by SSDs (red lines in Figs. 6a and $6 \mathrm{~b}$ ). These positional and strength preferences are not found before LTWE2 events (black lines in Figs. 6a and 6b). At positive lags, the centroid is displaced farther off of the pole and the vortex loses size and strength in wave events followed by SSDs with respect to those not followed by SSWs, as expected. No significant differences are found in the evolution of the vortex's aspect ratio (Fig. 6c). These results suggest that the vortex is preferentially strong and centered on the pole for tropospheric wave- 1 events to be followed by SSDs, and therefore puts the spotlight on the stratospheric state to determine whether or not an LTWE will be followed by an SSD.

\section{b. Are SSDs preceded by lower tropospheric wave events?}

We next address the problem from the opposite perspective: How many SSD events are preceded by anomalously strong lower tropospheric wave events? 

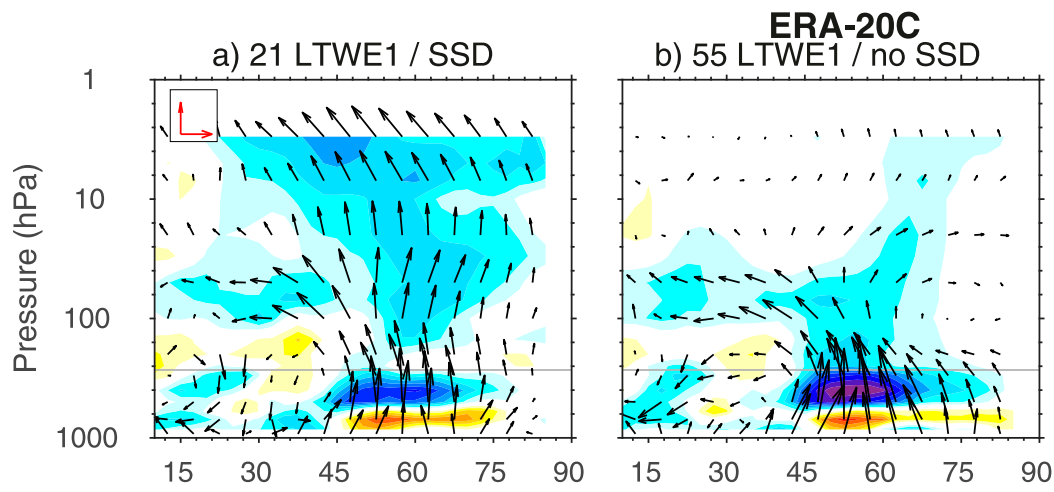

c) LTWE1 / events difference

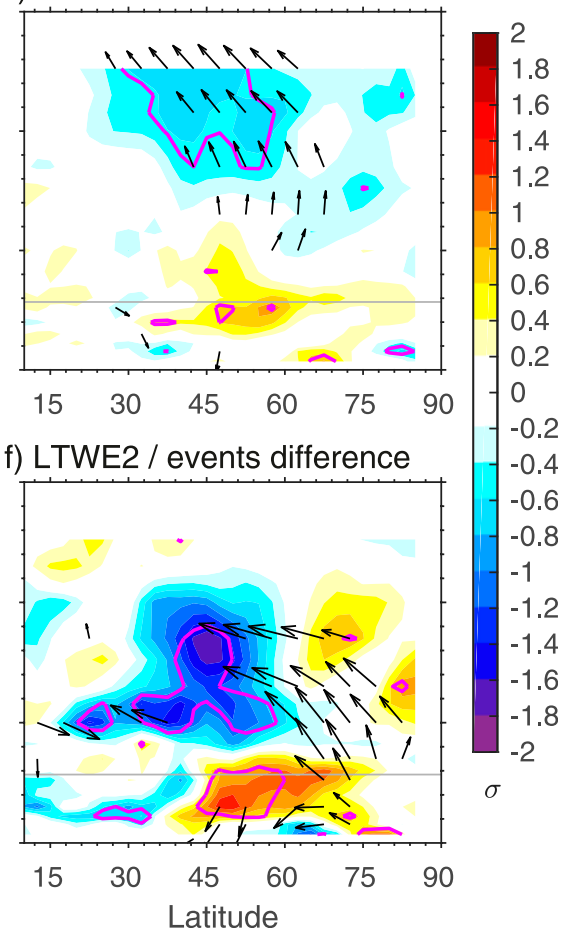

FIG. 4. As in Fig. 3, but for ERA-20C.

(We use here the same 10-day time scale). Table 2 presents the corresponding statistics. As in the previous subsection, the numbers are very similar between WACCM and ERA-20C: 37\% of all SSDs are preceded by LTWEs in the climate model, and $33 \%$ in the reanalysis. Our results are consistent with ERAInterim (BA17), as well as with a recent analysis of over 1500 years of climate model runs where $31 \%$ of all simulated SSWs were preceded by LTWEs (White et al. 2019). Sorting the stratospheric warmings as a function of the dominant wavenumber (see section $3 \mathrm{~b})$, we find that $38 \%(40 \%)$ of SSD1 events are preceded by LTWE1 in WACCM (ERA-20C), while $28 \%(29 \%)$ of SSD2 events are preceded by LTWE2. Note that SSD1 (SSD2) events represent 53\% (30\%) of all SSDs in WACCM, and 47\% (18\%) in ERA$20 \mathrm{C}$.

The standardized anomalies of $F_{z}(s=1+2)^{2}$ in the stratosphere present values over $2 \sigma$ in the \pm 5 days of the SSW central date, simultaneously with large wind deceleration anomalies (Figs. 7a,d). This is evidence of the positive feedback internal to the stratosphere between

\footnotetext{
${ }^{2} F_{z}(s=1+2)$ is normalized by the standard deviation of the sum of the wave- 1 and wave- 2 components of $F_{z}$.
}

upward wave activity and the mean flow that leads to SSDs (e.g., Sjoberg and Birner 2014). The composites show much weaker anomalies in the troposphere, slightly over $0.5 \sigma$ in WACCM and even weaker in ERA-20C (Figs. 7a,d). From this perspective, the amplification of $F_{z}$ in the stratosphere for the majority of events can hardly be attributed to a strong amplification in the tropospheric wave sources.

Sorting the SSDs regarding the dominant wavenumber reveals that SSD1 events have a stronger tropospheric signal of wave activity than SSD2 events, both in WACCM and ERA-20C (middle and right panels of Fig. 7), with peak anomalies reaching $1 \sigma$ in the 10 days prior to the stratospheric event (Figs. 7b,e). Given the relatively large sample of SSDs we are working with in both datasets, we can analyze in detail the evolution of the upward wave activity according to whether or not they were preceded by LTWEs. Focusing on SSD1 events first, we see in Fig. 8 that the evolutions of both the upward wave activity in the stratosphere and the deceleration anomalies are very similar in both type of events (i.e., preceded or not by LTWEs). One difference is that SSDs preceded by LTWEs have a peak in upward wave activity anomalies lower in the stratosphere than those SSDs not preceded by LTWE. But the main differences take place in the troposphere, where there is a much stronger wave activity signal if the SSDs are 
WACCM

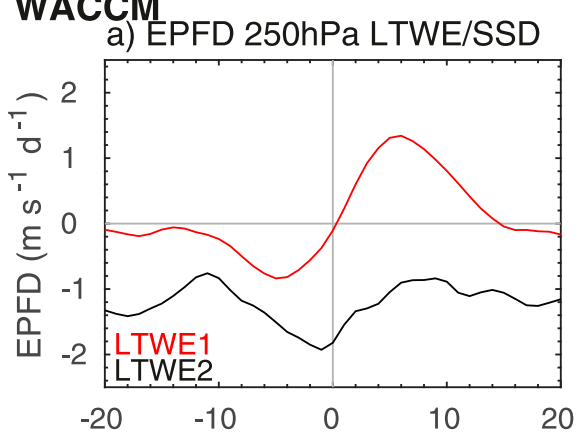

ERA-20C

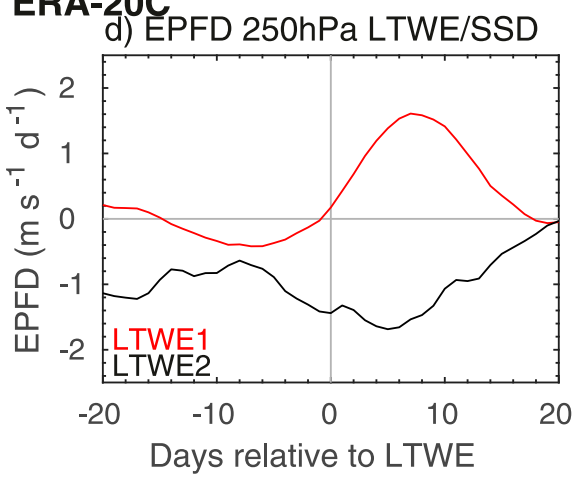

b) EPFD 250hPa LTWE/no SSD

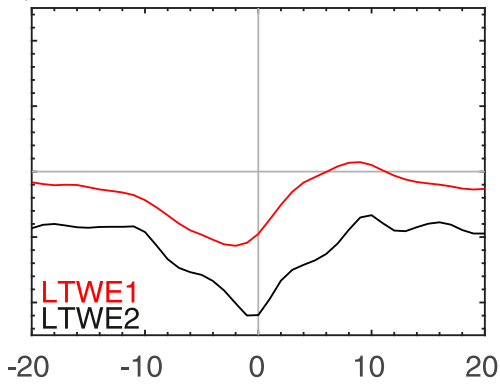

e) EPFD 250hPa LTWE/no SSD

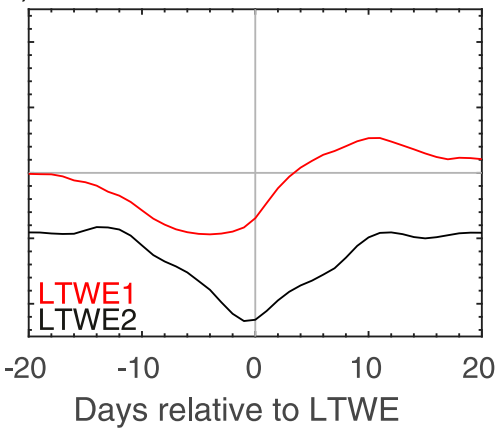

c) EPFD 250hPa LTWE/diff

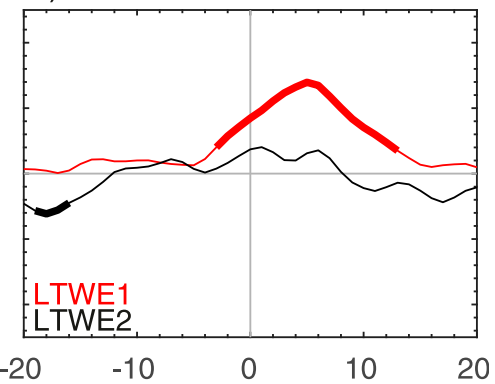

f) EPFD 250hPa LTWE/diff

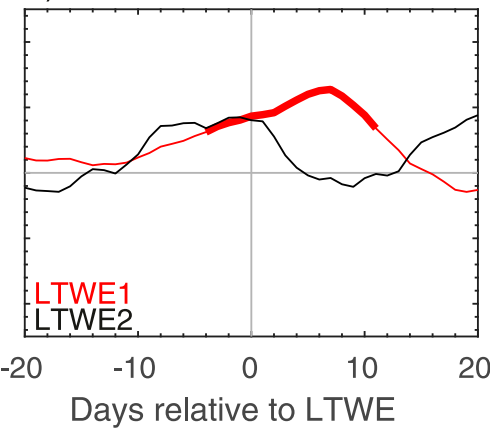

FIG. 5. Time evolution of the EP flux divergence (EPFD) (net values, no anomalies) at $250 \mathrm{hPa}$ around the LTWE central date. EPFD of the zonal harmonic $s=1$ is displayed for LTWE1 (red line) and of zonal harmonic $s=2$ for LTWE2 (black lines). (left),(middle) Composite evolution for LTWEs followed and not followed by SSDs, respectively, and (right) the difference with thick lines highlighting the statistically significant values (Student's $t$ test for the difference of means, $\alpha=0.05$ ). Results for (top) WACCM and (bottom) ERA20C are shown.

preceded by LTWEs (Figs. 8a,c). Therefore, the composite signal of wave fluxes in the troposphere preceding SSDs (particularly SSD1 events) evident in Figs. 7b and 7 e comes for the most part from a smaller subset of events (38\%) with substantial tropospheric wave fluxes (LTWEs), while the remainder of SSD1 events have quite small lower tropospheric fluxes. However, even in the events not linked to tropospheric wave events there are anomalies over one standard deviation in the upper troposphere, which could be related to recent findings on the relation between tropospheric synoptic events and lower stratospheric heat flux anomalies (Attard and Lang 2019). This happens both in WACCM and ERA-20C.

Figure 9 displays the corresponding panels for SSD2 events. The wave activity anomalies in the upper troposphere in cases not preceded by LTWEs are much weaker for SSD2 than SSD1 events in WACCM (cf. Figs. $9 \mathrm{~b}$ and $8 \mathrm{~b}$ ), perhaps suggesting that the connection with tropospheric dynamics is weaker for split than for displacement SSDs in the model. In ERA-20C this is different, standardized anomalies in the upper troposphere are quite similar in SSD1 and SSD2 events not preceded by LTWEs (cf. Figs. 9d and 8d). Similar to SSD1 events, the peak in wave activity anomalies happens lower in the stratosphere in SSD2 events that are preceded by LTWEs. This suggests that in cases for which there is LTWE, the wave activity propagates upward, grows and peaks/reaches maximum amplitude a bit lower in the stratosphere. But a similar message is conveyed here: The main difference between stratospheric events preceded and not preceded by LTWEs happens in the troposphere.

The wave- 1 source signal that appeared above the tropopause after LTWE1 events followed by SSDs (Figs. 5a,c) shows up as well when compositing as a function of SSD1 events (Fig. 10). The important aspect that Fig. 10 introduces is that this wave- 1 source signal is linked to the occurrence of SSD1 events regardless of whether or not they are preceded by LTWEs. This would suggest that processes internal to the stratosphere are playing an important role here.

We next analyze the vortex moment diagnostics in the midstratosphere $(850 \mathrm{~K})$. Figure 11 shows the composite evolution of the anomalies of the latitude of the centroid, area, and aspect ratio for SSD1 and SSD2 events. For the vortex centroid and area (left and middle panels), there is a clear cycle going from negative to positive lags where the vortex moves away from the pole 
WACCM

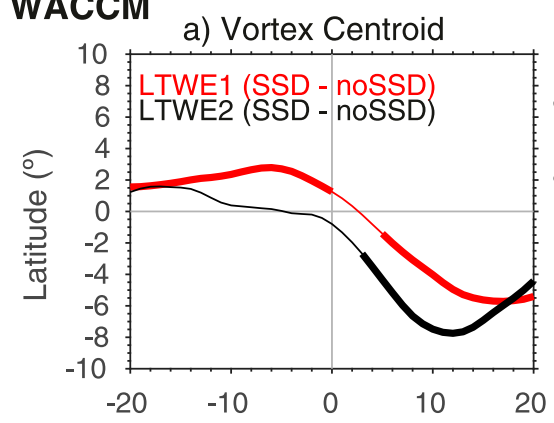

ERA-20C

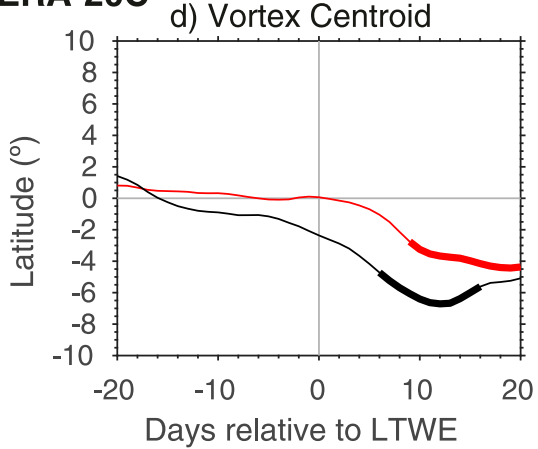

b) Vortex Area

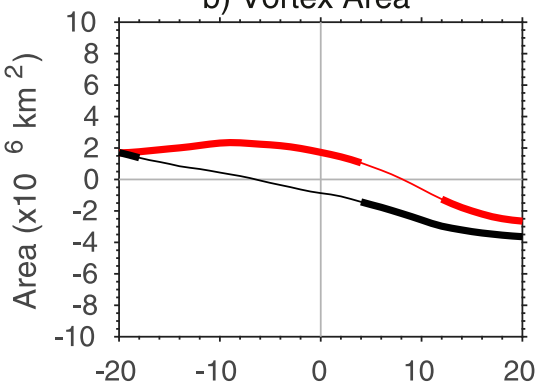

e) Vortex Area

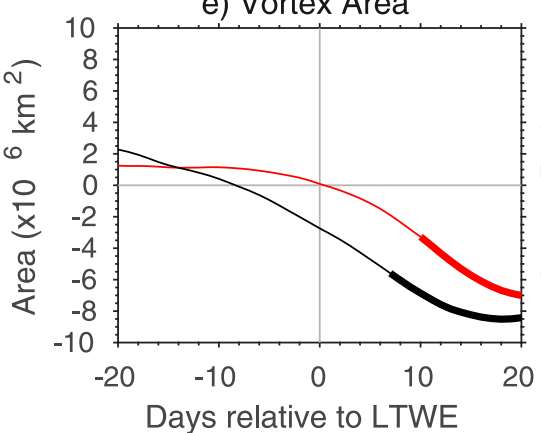

c) Vortex Aspect Ratio
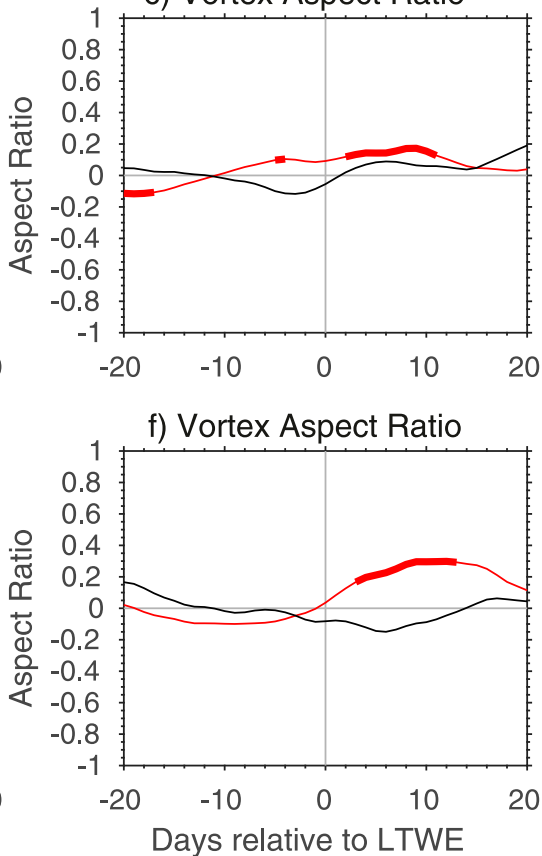

FIG. 6. Composite evolution around LTWE central dates of anomalies of elliptical diagnostics at 850 K: (a),(d) latitude of the vortex centroid, (b),(e) vortex area, and (c),(f) vortex aspect ratio, for (top) WACCM and (bottom) ERA-20C. Red (black) lines indicate the composite difference between LTWE1 (LTWE2) followed and not followed by SSDs, with thick lines highlighting the statistically significant values (Student's $t$ test for the difference of means, $\alpha=0.05$ ).

and shrinks. This is what is expected during the life cycle of sudden warmings. Looking into what happens at negative lags in more detail, we find some differences in the statistical significance of the signals between WACCM and ERA-20C. In the climate model the vortex centroid is significantly located $2^{\circ}$ to the north before SSD1 events (Fig. 11a), but in the reanalysis this is not the case (Fig. 11d). On the other hand, the reanalysis shows a significantly stronger and wider vortex up until $\sim 15$ days before both SSD1 and SSD2 events (Fig. 11e), as well as an elongated vortex before SSD2 events (expected since SSD2 will be dominated by split events) (Fig. 11f); but these signals are absent in the model. Generally, Fig. 11 shows a wider vortex with its center located closer to the pole before SSD1 events. To the extent to which SSD1 events will be dominated by displacement sudden warmings (Charlton and Polvani 2007), these results are fairly consistent with previous studies (e.g., Albers and Birner 2014). And importantly, isolating the SSD events that are preceded and not preceded by LTWEs does not change statistically significantly our results (see Fig. S1 in the online supplemental material). However, there is a lack of consistency between WACCM and ERA-20C for these vortex moment diagnostics that suggests that preconditioning signals are not very robust.

\section{Discussion and conclusions}

We have used 240 years from historical runs with the chemistry-climate model WACCM, and the twentiethcentury reanalysis ERA-20C to analyze the relation between anomalous lower tropospheric wave events (LTWEs) and the occurrence of sudden stratospheric deceleration (SSD) events, from which sudden stratospheric warmings defined as wind reversals at $60^{\circ} \mathrm{N}$ and $10 \mathrm{hPa}$ are a subset. Our results robustly show the following:

- only $\sim 20 \%$ of all LTWEs are followed by an SSD, and - only $\sim 1 / 3$ of all SSDs are preceded by an LTWE.

These results are not very sensitive to the specific thresholds used in the definitions of both LTWEs and SSDs (not shown).

One might argue that a threshold of (or around) $2 \sigma$ for the definition of LTWEs is too restrictive, and that SSDs may be more directly linked to anomalous situations in the troposphere of moderate intensity. To check this point, we have selected moderate LTWEs (mLTWEs) in WACCM, defined as those events where the anomalies of upward EP flux are larger than $1 \sigma$ but stay lower than $2 \sigma$. The percentages of 
TABLE 2. Number of SSDs preceded and not preceded by LTWEs.

\begin{tabular}{|c|c|c|c|c|c|c|}
\hline & & SSD1 & SSD2 & SSD1\&2 & SSDww & All SSD \\
\hline \multirow[t]{4}{*}{ WACCM } & Preceded by LTWE1 & $39(38 \%)$ & $2(6 \%)$ & $0(-)$ & $13(21 \%)$ & $54(27 \%)$ \\
\hline & Preceded by LTWE2 & $6(6 \%)$ & $9(28 \%)$ & $0(-)$ & $5(8 \%)$ & $20(10 \%)$ \\
\hline & Not preceded by LTWE & $61(58 \%)$ & $27(66 \%)$ & $0(-)$ & $44(71 \%)$ & $126(63 \%)$ \\
\hline & Total & 106 & 32 & 0 & 62 & 200 \\
\hline \multirow[t]{4}{*}{ ERA-20C } & Preceded by LTWE1 & $18(40 \%)$ & $1(6 \%)$ & $0(0 \%)$ & $4(13 \%)$ & $23(24 \%)$ \\
\hline & Preceded by LTWE2 & $1(2 \%)$ & $5(29 \%)$ & $1(33 \%)$ & $1(3 \%)$ & $8(8 \%)$ \\
\hline & Not preceded by LTWE & $26(58 \%)$ & $11(65 \%)$ & $2(67 \%)$ & $25(83 \%)$ & $64(67 \%)$ \\
\hline & Total & 45 & 17 & 3 & 30 & 95 \\
\hline
\end{tabular}

mLTWEs followed by SSDs, and of SSDs preceded by mLTWEs, are remarkably similar to our results with extreme LTWEs (see Tables S1 and S2 in the supplemental material). This indicates that the probability of occurrence of SSDs does not depend on the strength of the tropospheric wave activity anomalies, and further highlights the weak statistical connection between SSDs and anomalous wave events in the troposphere.

The consistency found between the model and the twentieth-century reanalysis confirms previous results with the shorter record of ERA-Interim (Birner and
Albers 2017), and provides statistically significant evidence that the anomalous amplification of wave activity in the stratosphere that drives SSDs is in most cases not due to an anomalous amplification of the waves in the source region (i.e., the lower troposphere). This is consistent with idealized model experiments (e.g., Christiansen 1999; Scott and Polvani 2004, 2006; Matthewman and Esler 2011; Esler and Matthewman 2011; Sjoberg and Birner 2014; Jucker 2016; de la Cámara et al. 2017; Lindgren et al. 2018), and demonstrated here in reanalysis and state-of-the-art chemistry climate model simulations (see also White et al. 2019).
WACCM

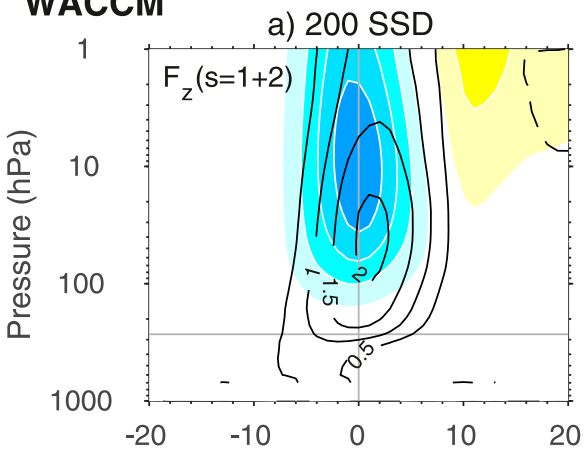

ERA-20C

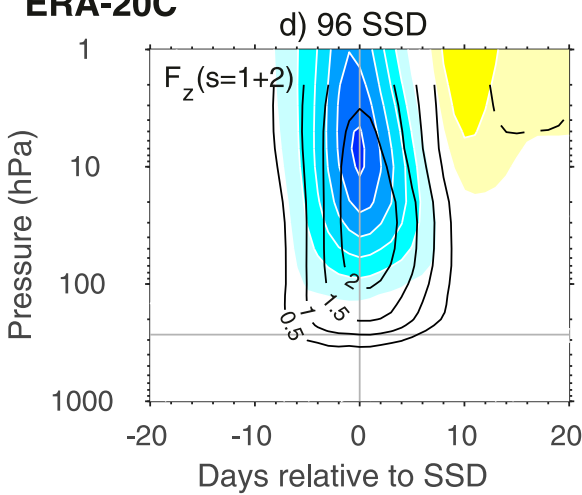

b) 106 SSD1

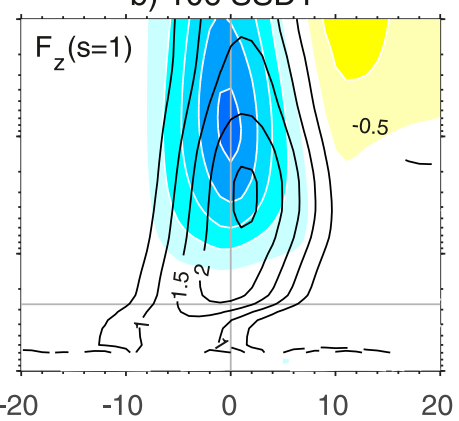

c) 32 SSD2

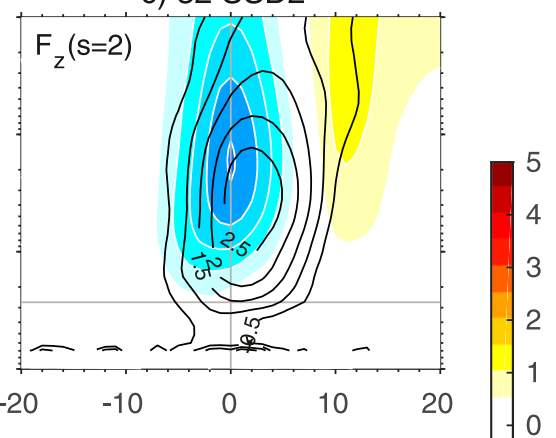

3

2 1 (1) e) 45 SSD1

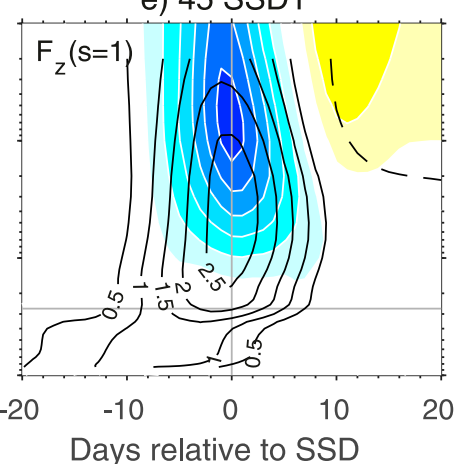

f) $17 \mathrm{SSD} 2$

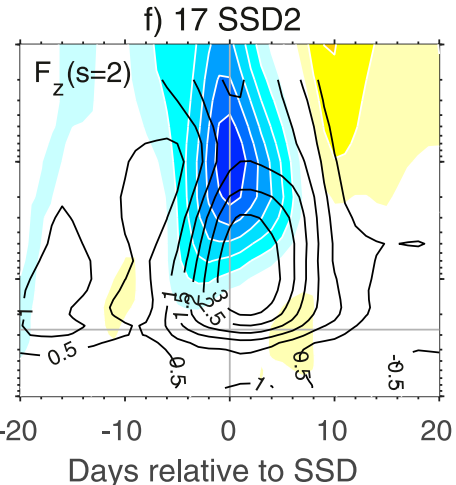


WACCM

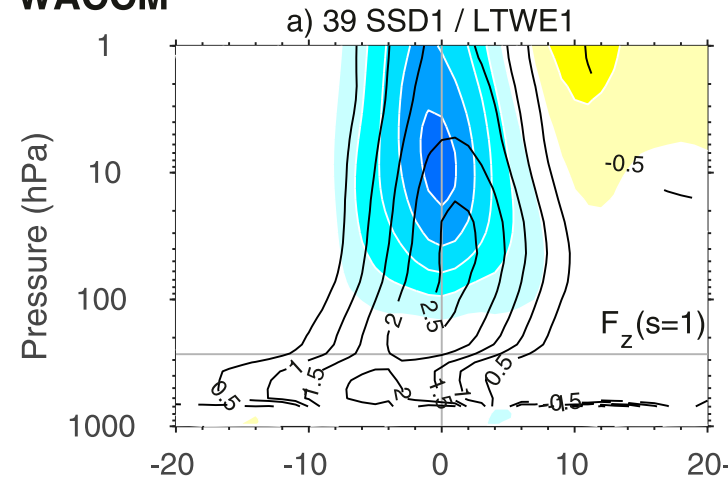

ERA-20C

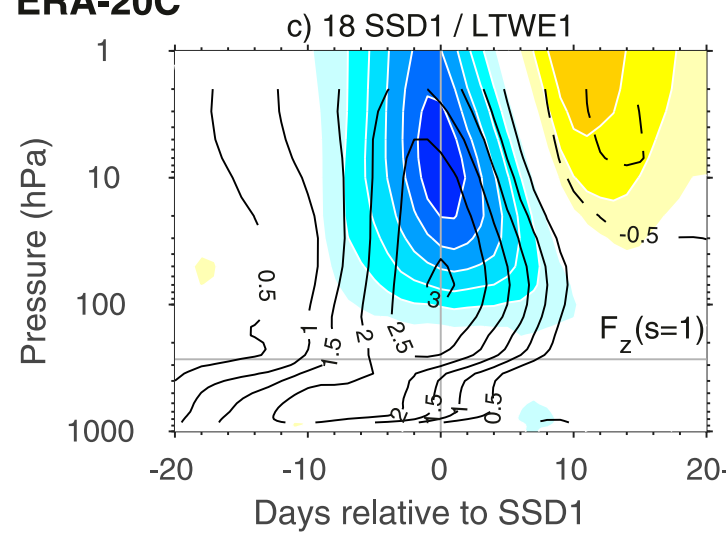

b) 61 SSD1 / no LTWE

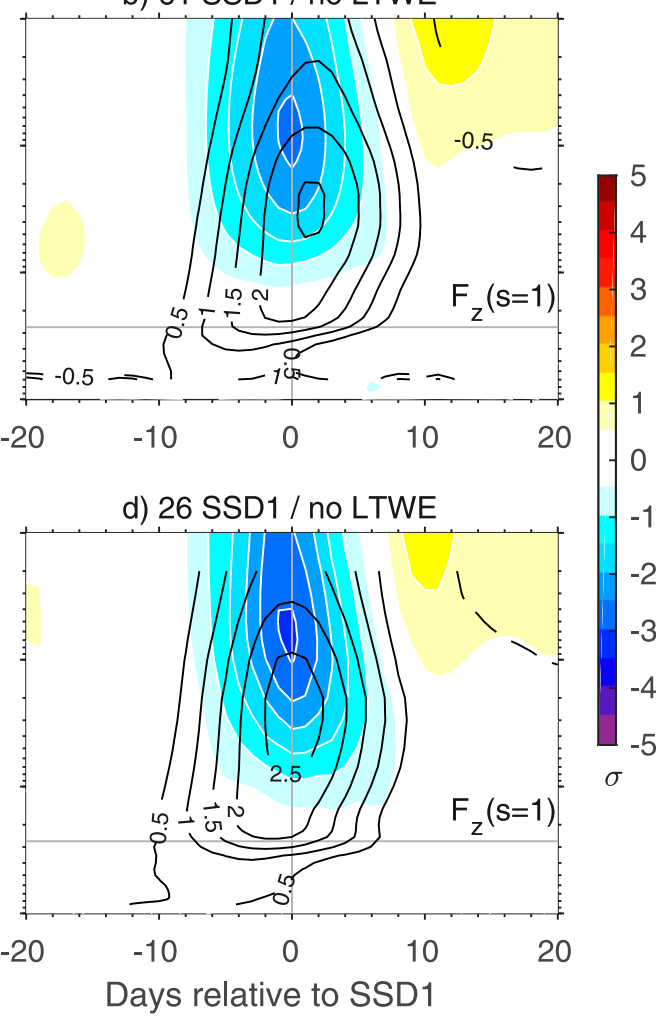

FIG. 8. Composite evolution, as a function of lag and pressure level, for (a),(c) SSD1 preceded by LTWE1 and (b),(d) SSD1 not preceded by LTWEs, in (top) WACCM and (bottom) ERA-20C. The upward EP flux anomaly [for zonal wavenumber $1 F_{z}(s=1)$ ] is shown in black contours, and the anomalies of the 10-day integrated wind tendency in colors. All anomalies are standardized [i.e., high values indicate high statistical significance ( $z$ score)]. Horizontal gray lines mark the approximate tropopause level $(\sim 270 \mathrm{hPa})$.

If anomalous tropospheric wave activity does not represent the main cause of the sudden rise of stratospheric upward EP fluxes, which process does? The answers that previous research offers give a primary role to the stratospheric circulation. Birner and Albers (2017) argued that the climatological wave activity in the troposphere is around one order of magnitude larger than in the stratosphere, so it may well be enough to tap into this reservoir below for the stratosphere to develop an SSW (see SSD composites of the net wave fluxes-no anomalies-in Figs. S2 and S3 in the supplemental material). This can be achieved with lower stratospheric configurations that allow extra penetration of the tropospheric wave activity (Chen and Robinson 1992), but also with a vortex geometry that favors the focusing of wave activity toward high latitudes (McIntyre 1982). Although it has been proven hard to diagnose (Albers and Birner 2014; Domeisen et al. 2018b), resonance is another process explaining rapid wave amplification in the stratosphere linked to SSWs (Clark 1974; Tung and Lindzen 1979; Plumb 1981; Esler and Scott 2005; Matthewman and Esler 2011).
Within this context, we have employed vortex moment diagnostics and shown that the vortex is generally wider and the center is located closer to the pole before wave-1 LTWEs (LTWE1) that are followed by SSDs (Fig. 6), a configuration found to precede wave-1 SSDs (SSD1) in a composite sense, regardless of whether they are or not connected to LTWEs (Fig. 11). We have also found that LTWE1 followed by SSD1 events are generally accompanied by a wave- 1 source just above the tropopause (Fig. 5), something found as well for the composite of all SSD1 events (Fig. 10). The reason for this wave source region is unclear, and conditions for shear instability are hardly met in our composite analysis (not shown), which calls for further research.

Our results in Figs. 7-9 suggest that the majority of stratospheric events do not trace back to anomalously strong tropospheric wave forcing. One would then expect ERA-20C, which only includes assimilation of surface observations, to do poorly in simulating the correct timing of stratospheric events such as SSDs. We have compared event dates in ERA-20C to those found 


\section{WACCM}

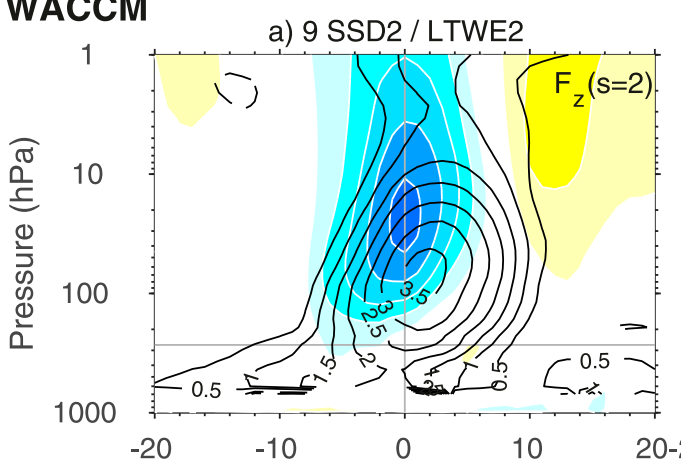

ERA-20C

c) 5 SSD2 / LTWE2

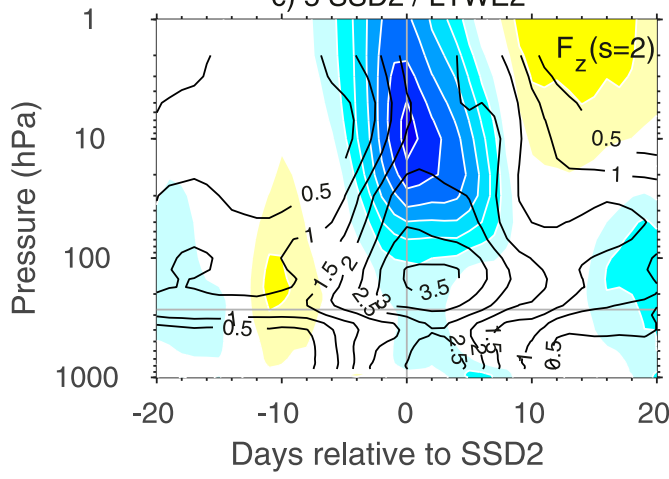

b) 21 SSD2 / no LTWE

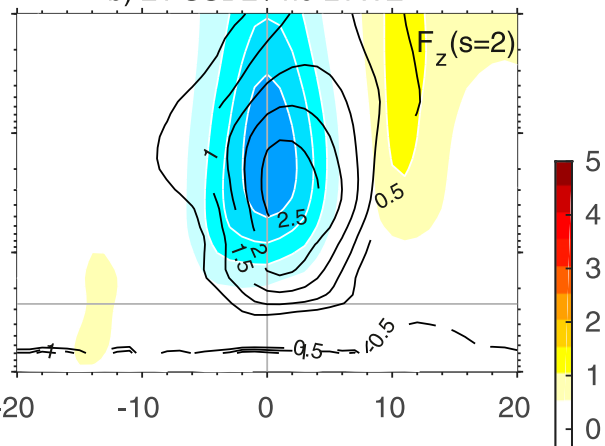

d) 11 SSD2 / no LTWE

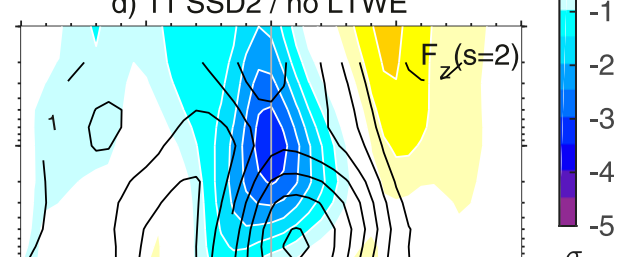

$\sigma$

FIG. 9. As in Fig. 8, but for SSD2 events, and black contours showing anomalies of upward wave activity for zonal wavenumber $2\left[F_{z}(s=2)\right]$.

in ERA-Interim by BA17 (see Table S3 in the supplemental material) for the common period of 1979-2010. Surprisingly, 10 out of 27 SSDs found in ERA-Interim are reproduced to within 3 days by ERA-20C. Furthermore, for many of the remaining $17 \mathrm{SSD}$ events the evolution of the zonal mean zonal wind at $60^{\circ}$ and $10 \mathrm{hPa}$ is remarkably similar between ERA-20C and ERAInterim (not shown). Nevertheless, there are striking
WACCM

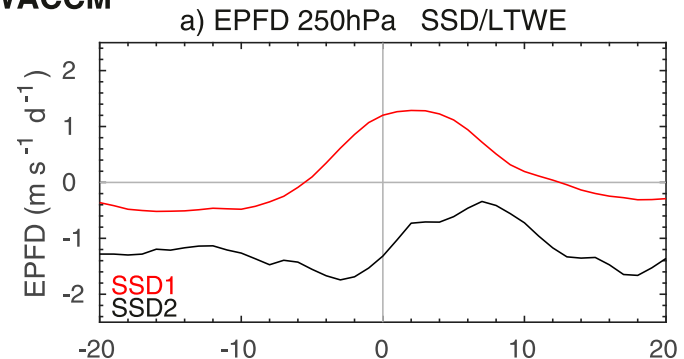

ERA-20C

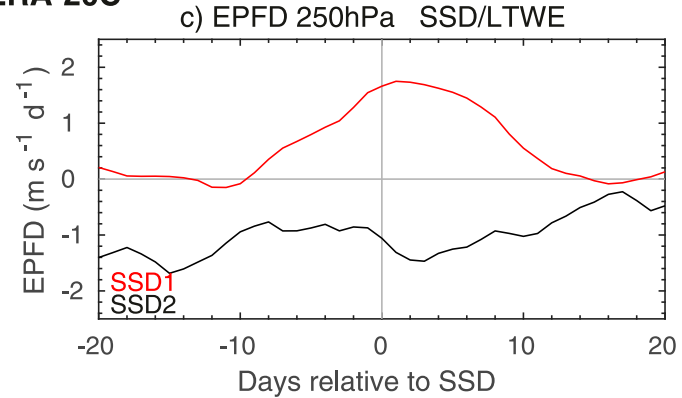

b) EPFD $250 \mathrm{hPa}$ SSD/no LTWE

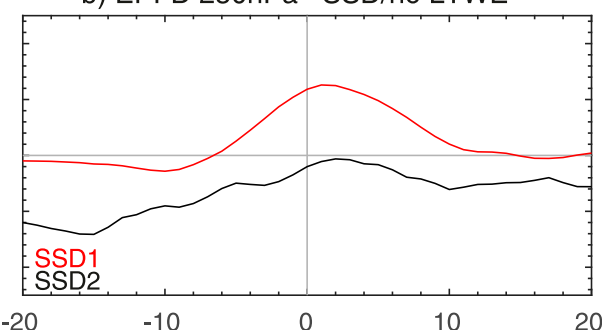

d) EPFD 250hPa SSD/no LTWE

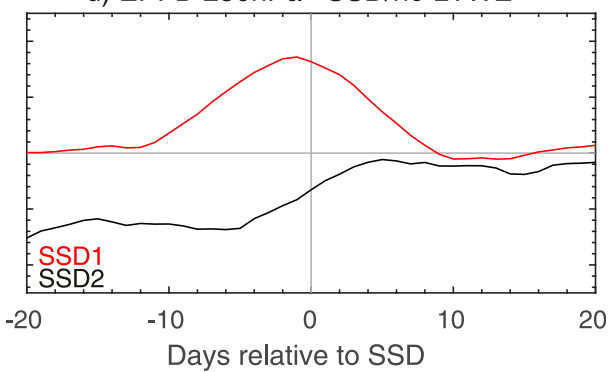

FIG. 10. As in Fig. 5, but for SSD events preceded and not by LTWEs. 
WACCM
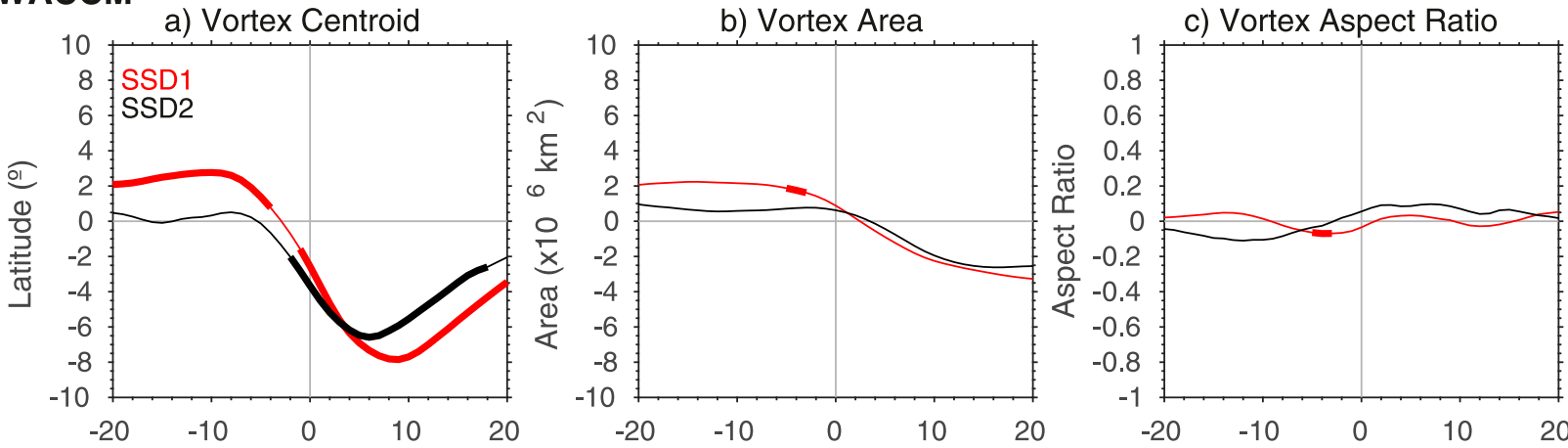

\section{ERA-20C}
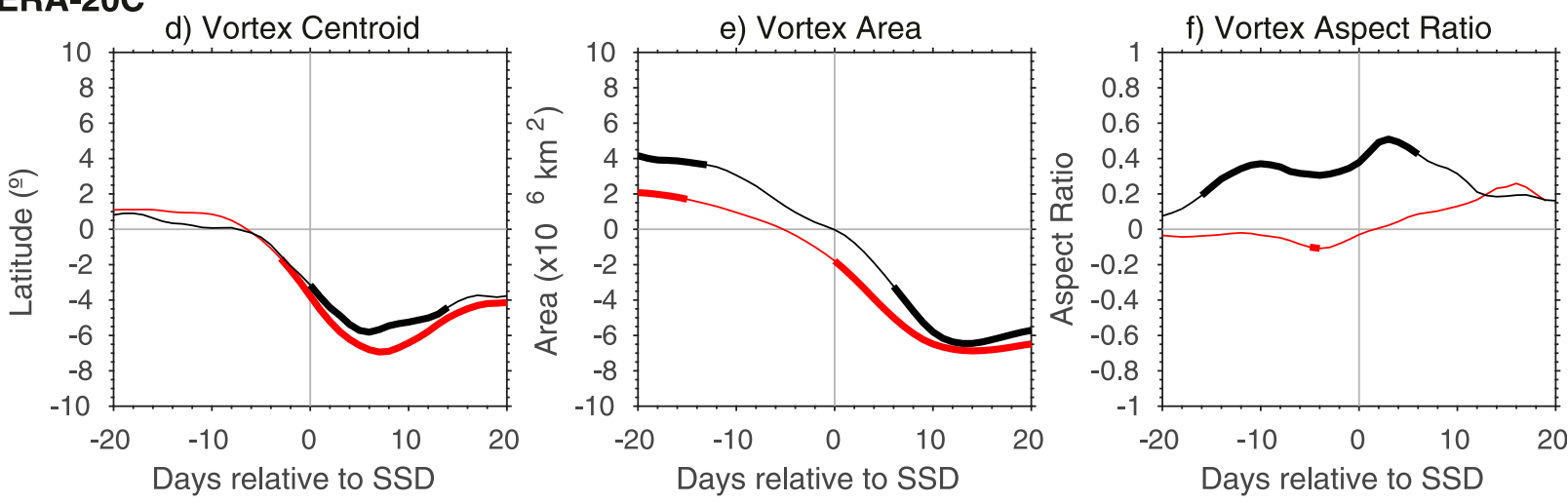

FIG. 11. Composite evolution around SSD central dates of anomalies of elliptical diagnostics at $850 \mathrm{~K}$ : (a),(d) latitude of the vortex centroid, (b),(e) vortex area, and (c),(f) vortex aspect ratio, for (top) WACCM and (bottom) ERA-20C. Red and black lines indicate the composite anomalies for all SSD1 and SSD2, respectively, with thick lines highlighting the statistically significant values (Student's $t$ test, $\alpha=0.05$ ).

counterexamples where the event is essentially missed completely in ERA-20C, such as the January 2009 SSW, which was previously interpreted to have been triggered by resonance (Albers and Birner 2014).

Is the knowledge of the near-surface wave fluxes and their correct evolution (likely to be the case in ERA20C) sufficient then to reproduce SSDs in many cases? First, note that 5 out of the subset of 10 common SSD events between the datasets are associated with an LTWE (see Table 1 in the supplemental material of BA17), which may indicate that these 5 events were indeed triggered by a pulse of anomalously strong upward wave flux from the lower troposphere. However, we also note that the stratospheric state prior to SSDs is essentially a function of the history of wave fluxes of tropospheric origin and their (nontrivial) interactions with the stratospheric mean flow. More or less constraining the evolution of lower tropospheric wave fluxes in ERA-20C will likely "nudge" the entire column toward the observed state. Furthermore, it is important to note that data assimilation (such as the 4DVar system used for ERA-20C) can be quite powerful in adjusting the flow field remote to the region of data input. For example, Compo et al. (2006) showed how assimilating a single surface measurement that is only $1 \mathrm{hPa}$ different from the model "first guess" produces a vertically deep response all the way up to at least $300 \mathrm{hPa}$. The implication is that data assimilation of surface observations, particularly those of surface pressure, has a far field effect that likely effectively constrains the wave flux evolution of the entire troposphere. Nevertheless, the fact that more than half of the observed SSDs between 1979 and 2010 are not captured in ERA-20C means that the nonlinearity of the positive wave-mean flow feedback leading to SSDs (and SSWs) may break the above mentioned effective constraints and thereby limit the predictability of SSDs.

The search for precursors of sudden stratospheric warmings is in good part motivated by the additional skill that these events provide in subseasonal to seasonal forecasts (e.g., Tripathi et al. 2015; Butler et al. 2019). In this regard, our findings suggest to look in more detail into lowermost stratospheric dynamics and vortex geometry. New diagnostics based on the position and geometry of the vortex, such as those developed in Lawrence and Manney (2018), have the potential to 
overcome the difficulty of interpreting longitudinally averaged quantities in largely nonzonal situations such as during stratospheric warmings.

Acknowledgments. The authors are thankful to three anonymous reviewers for their constructive comments, and to Marta Abalos and Blanca Ayarzagüena for helpful discussions. This work has been partially funded by the Spanish Ministry of Economy and Innovation grants "PaleoStrat" (CGL2015-69699) and "Steady" (CGL201783198-R). AdlC has been supported by the UCM research fellowship Personal posdoctoral de formación en docencia e investigación en los Departamentos de la UCM. TB acknowledges support by the U.S. National Science Foundation Climate Dynamics Program (Award 1643167). Data from ERA-20C are available at http://apps.ecmwf.int/ datasets/ and WACCM output are available at https:// www2.acom.ucar.edu/gcm/ccmi-output and also upon request to the corresponding author.

\section{REFERENCES}

Albers, J. R., and T. Birner, 2014: Vortex preconditioning due to planetary and gravity waves prior to sudden stratospheric warmings. J. Atmos. Sci., 71, 4028-4054, https://doi.org/ 10.1175/JAS-D-14-0026.1.

Andrews, D. G., J. R. Holton, and C. B. Leovy, 1987: Middle Atmosphere Dynamics. Academic Press, 489 pp.

Attard, H. E., and A. L. Lang, 2019: Troposphere-stratosphere coupling following tropospheric blocking and extratropical cyclones. Mon. Wea. Rev., 147, 1781-1804, https://doi.org/ 10.1175/MWR-D-18-0335.1.

- R. Rios-Berrios, C. T. Guastini, and A. L. Lang, 2016: Tropospheric and stratospheric precursors to the January 2013 sudden stratospheric warming. Mon. Wea. Rev., 144, 13211339, https://doi.org/10.1175/MWR-D-15-0175.1.

Ayarzagüena, B., D. Barriopedro, J. M. Garrido-Perez, M. Abalos, A. de la Cámara, R. García-Herrera, N. Calvo, and C. Ordóñez, 2018: Stratospheric connection to the abrupt end of the 2016/2017 Iberian drought. Geophys. Res. Lett., 45, 12 639-12 646, https://doi.org/10.1029/2018GL079802.

Baldwin, M. P., and T. J. Dunkerton, 2001: Stratospheric harbingers of anomalous weather regimes. Science, 294, 581-584, https://doi.org/10.1126/science.1063315.

Barriopedro, D., and N. Calvo, 2014: On the relationship between ENSO, stratospheric sudden warmings, and blocking. J. Climate, 27, 4704-4720, https://doi.org/10.1175/JCLI-D-13-00770.1.

Birner, T., and J. R. Albers, 2017: Sudden stratospheric warmings and anomalous upward wave activity flux. SOLA, 13A , 8-12, https://doi.org/10.2151/sola.13a-002.

- D. W. J. Thompson, and T. G. Shepherd, 2013: Up-gradient eddy fluxes of potential vorticity near the subtropical jet. Geophys. Res. Lett., 40, 5988-5993, https://doi.org/10.1002/2013GL057728.

Butler, A. H., and L. M. Polvani, 2011: El Niño, La Niña, and stratospheric sudden warmings: A reevaluation in light of the observational record. Geophys. Res. Lett., 38, L13807, https:// doi.org/10.1029/2011GL048084.

—, D. J. Seidel, S. C. Hardiman, N. Butchart, T. Birner, and A. Match, 2015: Defining sudden stratospheric warmings. Bull.
Amer. Meteor. Soc., 96, 1913-1928, https://doi.org/10.1175/ BAMS-D-13-00173.1.

- and Coauthors, 2019: Sub-seasonal predictability and the stratosphere. Sub-Seasonal to Seasonal Prediction: The Gap Between Weather and Climate Forecasting, A. W. Robertson and F. Vitart, Eds., Elsevier, 223-241, doi:10.1016/ B978-0-12-811714-9.00011-5.

Charlton, A. J., and L. M. Polvani, 2007: A new look at stratospheric sudden warmings. Part I: Climatology and modeling benchmarks. J. Climate, 20, 449-469, https://doi.org/10.1175/ JCLI3996.1.

Charney, J. G., and M. E. Stern, 1962: On the stability of internal baroclinic jets in a rotating atmosphere. J. Atmos. Sci., 19, 159-172, https://doi.org/10.1175/1520-0469(1962)019<0159: OTSOIB $>2.0 . \mathrm{CO} ; 2$.

Chen, M., C. R. Mechoso, and J. D. Farrara, 2001: Interannual variations in the stratospheric circulation with a perfectly steady troposphere. J. Geophys. Res., 106, 5161-5172, https:// doi.org/10.1029/2000JD900624.

Chen, P., and W. A. Robinson, 1992: Propagation of planetary waves between the troposphere and stratosphere. J. Atmos. Sci., 49, 2533-2545, https://doi.org/10.1175/1520-0469(1992) 049<2533:POPWBT>2.0.CO;2.

Christiansen, B., 1999: Stratospheric vacillations in a general circulation model. J. Atmos. Sci., 56, 1858-1872, https://doi.org/ 10.1175/1520-0469(1999)056<1858:SVIAGC $>2.0 . C O ; 2$.

Clark, J. H. E., 1974: Atmospheric response to the quasi-resonant growth of forced planetary waves. J. Meteor. Soc. Japan, 52, 143-162, https://doi.org/10.2151/jmsj1965.52.2_143.

Colucci, S. J., and M. E. Kelleher, 2015: Diagnostic comparison of tropospheric blocking events with and without sudden stratospheric warming. J. Atmos. Sci., 72, 2227-2240, https:// doi.org/10.1175/JAS-D-14-0160.1.

Compo, G. P., J. S. Whitaker, and P. D. Sardeshmukh, 2006: Feasibility of a 100-year reanalysis using only surface pressure data. Bull. Amer. Meteor. Soc., 87, 175-190, https://doi.org/ 10.1175/BAMS-87-2-175.

de la Cámara, A., J. R. Albers, T. Birner, R. R. Garcia, P. Hitchcock, D. E. Kinnison, and A. K. Smith, 2017: Sensitivity of sudden stratospheric warmings to previous stratospheric conditions. J. Atmos. Sci., 74, 2857-2877, https:// doi.org/10.1175/JAS-D-17-0136.1.

, M. Abalos, and P. Hitchcock, 2018a: Changes in stratospheric transport and mixing during sudden stratospheric warmings. J. Geophys. Res., 123, 3356-3373, https://doi.org/10.1002/ 2017JD028007.

$[,-,-$, N. Calvo, and R. R. Garcia, 2018b: Response of Arctic ozone to sudden stratospheric warmings. Atmos. Chem. Phys., 18, 16499-16513, https://doi.org/10.5194/acp-18-16499-2018.

Domeisen, D. I. V., C. I. Garfinkel, and A. H. Butler, 2018a: The teleconnection of El Niño Southern Oscillation to the stratosphere. Rev. Geophys., 57, 5-47, https://doi.org/10.1029/ 2018RG000596.

_, O. Martius, and B. Jiménez-Esteve, 2018b: Rossby wave propagation into the Northern Hemisphere stratosphere: The role of zonal phase speed. Geophys. Res. Lett., 45, 2064-2071, https://doi.org/10.1002/2017GL076886.

Esler, J. G., and R. K. Scott, 2005: Excitation of transient Rossby waves on the stratospheric polar vortex and the barotropic sudden warming. J. Atmos. Sci., 62, 3661-3682, https://doi.org/ 10.1175/JAS3557.1.

, and N. J. Matthewman, 2011: Stratospheric sudden warmings as self-tuning resonances. Part II: Vortex displacement events. 
J. Atmos. Sci., 68, 2505-2523, https://doi.org/10.1175/JAS-D11-08.1.

Garcia, R. R., A. K. Smith, D. E. Kinnison, A. de la Cámara, and D. J. Murphy, 2017: Modification of the gravity wave parameterization in the Whole Atmosphere Community Climate Model: Motivation and results. J. Atmos. Sci., 74, 275-291, https://doi.org/10.1175/JAS-D-16-0104.1.

Garfinkel, C. I., A. H. Butler, D. W. Waugh, M. M. Hurwitz, and L. M. Polvani, 2012: Why might stratospheric sudden warmings occur with similar frequency in El Niño and La Niña winters? J. Geophys. Res., 117, D19106, https://doi.org/ 10.1029/2012JD017777.

, C. Schwartz, D. I. V. Domeisen, S.-W. Son, A. H. Butler, and I. P. White, 2018: Extratropical atmospheric predictability from the quasi-biennial oscillation in subseasonal forecast models. J. Geophys. Res. Atmos., 123, 7855-7866, https:// doi.org/10.1029/2018JD028724.

Gerber, E. P., and P. Martineau, 2018: Quantifying the variability of the annular modes: Reanalysis uncertainty vs. sampling uncertainty. Atmos. Chem. Phys., 18, 17 099-17117, https:// doi.org/10.5194/acp-18-17099-2018.

Hitchcock, P., 2019: On the value of reanalyses prior to 1979 for dynamical studies of stratosphere-troposphere coupling. Atmos. Chem. Phys., 19, 2749-2764, https://doi.org/10.5194/acp19-2749-2019.

— , and P. H. Haynes, 2016: Stratospheric control of planetary waves. Geophys. Res. Lett., 43, $11884-11892$, https://doi.org/ 10.1002/2016GL071372.

Holton, J. R., and C. Mass, 1976: Stratospheric vacillation cycles. J. Atmos. Sci., 33, 2218-2225, https://doi.org/10.1175/15200469(1976)033<2218:SVC > 2.0.CO;2.

Hoskins, B., 1983: Modelling of the transient eddies and their feedback on the mean flow. Large-Scale Dynamical Processes in the Atmosphere, B. J. Hoskins and R. Pearce, Eds., Academic Press, 169-199.

Jucker, M., 2016: Are sudden stratospheric warmings generic? Insights from an idealized GCM. J. Atmos. Sci., 73, 5061-5080, https://doi.org/10.1175/JAS-D-15-0353.1.

Kidston, J., A. A. Scaife, S. C. Hardiman, D. M. Mitchell, N. Butchart, M. P. Baldwin, and L. J. Gray, 2015: Stratospheric influence on tropospheric jet streams, storm tracks and surface weather. Nat. Geosci., 8, 433-440, https://doi.org/10.1038/ngeo2424.

Labitzke, K., 1977: Interannual variability of the winter stratosphere in the Northern Hemisphere. Mon. Wea. Rev., 105, 762-770, https://doi.org/10.1175/1520-0493(1977)105<0762: IVOTWS $>2.0 . \mathrm{CO} ; 2$.

, 1978: On the different behavior of the zonal harmonic height waves 1 and 2 during the winters 1970/71 and 1971/72. Mon. Wea. Rev., 106, 1704-1713, https://doi.org/10.1175/15200493(1978)106<1704:OTDBOT>2.0.CO;2.

_ 1981: Stratospheric-mesospheric midwinter disturbances: A summary of observed characteristics. J. Geophys. Res., 86, 9665-9678, https://doi.org/10.1029/JC086iC10p09665.

Lawrence, Z. D., and G. L. Manney, 2018: Characterizing stratospheric polar vortex variability with computer vision techniques. J. Geophys. Res. Atmos., 123, 1510-1535, https:// doi.org/10.1002/2017JD027556.

Lindgren, E. A., A. Sheshadri, and R. A. Plumb, 2018: Sudden stratospheric warming formation in an idealized general circulation model using three types of tropospheric forcing. J. Geophys. Res., 123, $10125-10$ 139, https://doi.org/10.1029/2018JD028537.

Marsh, D. R., M. J. Mills, D. E. Kinnison, J.-F. Lamarque, N. Calvo, and L. M. Polvani, 2013: Climate change from 1850 to 2005 simulated in CESM1(WACCM). J. Climate, 26, 7372 7391, https://doi.org/10.1175/JCLI-D-12-00558.1.

Marshall, A. G., and A. A. Scaife, 2010: Improved predictability of stratospheric sudden warming events in an atmospheric general circulation model with enhanced stratospheric resolution. J. Geophys. Res., 115, D16114, https://doi.org/10.1029/ 2009JD012643.

Martineau, P., and S.-W. Son, 2015: Onset of circulation anomalies during stratospheric vortex weakening events: The role of planetary-scale waves. J. Climate, 28, 7347-7370, https:// doi.org/10.1175/JCLI-D-14-00478.1.

_, G. Chen, S.-W. Son, and J. Kim, 2018a: Lower-stratospheric control of the frequency of sudden stratospheric warming events. J. Geophys. Res., 123, 3051-3070, https://doi.org/ 10.1002/2017JD027648.

_ J. S. Wright, N. Zhu, and M. Fujiwara, 2018b: Zonal-mean data set of global atmospheric reanalyses on pressure levels. Earth Syst. Sci. Data, 10, 1925-1941, https://doi.org/10.5194/ essd-10-1925-2018.

Martius, O., L. M. Polvani, and H. C. Davies, 2009: Blocking precursors to stratospheric sudden warming events. $G e$ ophys. Res. Lett., 36, L14806, https://doi.org/10.1029/ 2009GL038776.

Matsuno, T., 1971: A dynamical model of the stratospheric sudden warming. J. Atmos. Sci., 28, 1479-1494, https://doi.org/10.1175/ 1520-0469(1971)028<1479:ADMOTS >2.0.CO;2.

Matthewman, N. J., and J. G. Esler, 2011: Stratospheric sudden warmings as self-tuning resonances. Part I: Vortex splitting events. J. Atmos. Sci., 68, 2481-2504, https://doi.org/10.1175/ JAS-D-11-07.1.

,-- , A. J. Charlton-Perez, and L. M. Polvani, 2009: A new look at stratospheric sudden warmings. Part III: Polar vortex evolution and vertical structure. J. Climate, 22, 1566-1585, https://doi.org/10.1175/2008JCLI2365.1.

McIntyre, M. E., 1982: How well do we understand the dynamics of stratospheric warmings? J. Meteor. Soc. Japan, 60, 37-65, https://doi.org/10.2151/JMSJ1965.60.1_37.

Melander, M. V., N. J. Zabusky, and A. S. Styczek, 1986: A moment model for vortex interactions of the two-dimensional Euler equations. Part 1. Computational validation of a Hamiltonian elliptical representation. J. Fluid Mech., 167, 95-115, https://doi.org/10.1017/S0022112086002744.

Newman, P. A., and J. E. Rosenfield, 1997: Stratospheric thermal damping times. Geophys. Res. Lett., 24, 433-436, https:// doi.org/10.1029/96GL03720.

Plumb, R. A., 1981: Instability of the distorted polar night vortex: A theory of stratospheric warmings. J. Atmos. Sci., 38, 2514-2531, https://doi.org/10.1175/1520-0469(1981) $038<2514$ :IOTDPN $>2.0$.CO;2.

Poli, P., and Coauthors, 2016: ERA-20C: An atmospheric reanalysis of the twentieth century. J. Climate, 29, 4083-4097, https://doi.org/10.1175/JCLI-D-15-0556.1.

Polvani, L. M., and D. W. Waugh, 2004: Upward wave activity flux as a precursor to extreme stratospheric events and subsequent anomalous surface weather regimes. J. Climate, 17, 3548-3554, https://doi.org/10.1175/1520-0442(2004)017<3548: UWAFAA $>2.0 . \mathrm{CO} ; 2$.

Scaife, A., and Coauthors, 2014: Skillful long range prediction of European and North American winters. Geophys. Res. Lett., 41, 2514-2519, https://doi.org/10.1002/2014GL059637.

— , and Coauthors, 2016: Seasonal winter forecasts and the stratosphere. Atmos. Sci. Lett., 17, 51-56, https://doi.org/ 10.1002/asl.598. 
Scott, R. K., and P. H. Haynes, 2000: Internal vacillations in stratosphere-only models. J. Atmos. Sci., 57, 3233-3250, https:// doi.org/10.1175/1520-0469(2000)057<3233:IVISOM>2.0.CO;2.

—, and L. M. Polvani, 2004: Stratospheric control of upward wave flux near the tropopause. Geophys. Res. Lett., 31, L02115, https://doi.org/10.1029/2003GL017965.

—, and - 2006: Internal variability of the winter stratosphere. Part I: Time-independent forcing. J. Atmos. Sci., 63, 27582776, https://doi.org/10.1175/JAS3797.1.

Sjoberg, J. P., and T. Birner, 2012: Transient tropospheric forcing of sudden stratospheric warmings. J. Atmos. Sci., 69, 3420 3432, https://doi.org/10.1175/JAS-D-11-0195.1.

_ and , 2014: Stratospheric wave-mean flow feedbacks and sudden stratospheric warmings in a simple model forced by upward wave activity flux. J. Atmos. Sci., 71, 4055-4071, https://doi.org/10.1175/JAS-D-14-0113.1.

Smith, A. K., 1983: Observation of wave-wave interactions in the stratosphere. J. Atmos. Sci., 40, 2484-2496, https://doi.org/ 10.1175/1520-0469(1983)040<2484:OOWWII >2.0.CO;2.

_ 1989: An investigation of resonant waves in a numerical model of an observed sudden stratospheric warming. J. Atmos. Sci., 46, 3038-3054, https://doi.org/10.1175/1520-0469(1989) 046<3038:AIORWI>2.0.CO;2.

_ 1992: Preconditioning for stratospheric sudden warmings: Sensitivity studies with a numerical model. J. Atmos. Sci., 49 1003-1019, https://doi.org/10.1175/1520-0469(1992)049<1003: PFSSWS $>2.0 . \mathrm{CO} ; 2$.

_ - J. C. Gille, and L. V. Lyjak, 1984: Wave-wave interactions in the stratosphere: Observations during quiet and active wintertime periods. J. Atmos. Sci., 41, 363-373, https://doi.org/ 10.1175/1520-0469(1984)041<0363:WIITSO > 2.0.CO;2.

Smith, K. L., and P. J. Kushner, 2012: Linear interference and the initiation of extratropical stratosphere-troposphere interactions. J. Geophys. Res., 117, D13107, https://doi.org/10. 1029/2012JD017587.

Thompson, D. W. J., M. P. Baldwin, and J. M. Wallace, 2002: Stratospheric connection to Northern Hemisphere wintertime weather: Implications for prediction. J. Climate, 15, 1421-1428, https:// doi.org/10.1175/1520-0442(2002)015<1421:SCTNHW>2.0.CO;2.

Tripathi, O. P., and Coauthors, 2015: The predictability of the extratropical stratosphere on monthly time-scales and its impact on the skill of tropospheric forecasts. Quart. J. Roy. Meteor. Soc., 141, 987-1003, https://doi.org/10.1002/qj.2432.

Tung, K. K., and R. S. Lindzen, 1979: A theory of stationary long waves. Part II: Resonant Rossby waves in the presence of realistic vertical shears. Mon. Wea. Rev., 107, 735-750, https://doi.org/ 10.1175/1520-0493(1979)107<0735:ATOSLW>2.0.CO;2.

Waugh, D. N. W., 1997: Elliptical diagnostics of stratospheric polar vortices. Quart. J. Roy. Meteor. Soc., 123, 1725-1748, https:// doi.org/10.1002/qj.49712354213.

White, I., C. I. Garfinkel, E. P. Gerber, M. Jucker, V. Aquila, and L. D. Oman, 2019: The downward influence of sudden stratospheric warmings: Association with tropospheric precursors. J. Climate, 32, 85-108, https://doi.org/10.1175/JCLI-D-18-0053.1.

Yoden, S., 1987: Dynamical aspects of stratospheric vacillations in a highly truncated model. J. Atmos. Sci., 44, 36833695, https://doi.org/10.1175/1520-0469(1987)044<3683: DAOSVI $>2.0 . \mathrm{CO} ; 2$. 